\title{
Economy and Environment of a Medieval Town Reflected in Wells Backfill in Písek, Bakaláře Square (South Bohemia, Czech Republic)
}

\author{
Tereza Šálkováa, b, c*, Petra Houfkováa, Jaroslav Jiřík ${ }^{\mathrm{b}}$, Lenka Kovačikováe, Jan Novák ${ }^{\mathrm{a}}$,

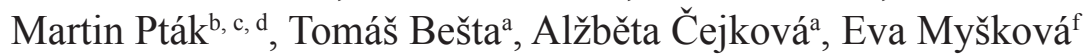 \\ ${ }^{a}$ University of South Bohemia, Faculty of Science, Na Zlaté stoce 3, Laboratory of Archaeobotany and Palaeoecology, 37005 České Budějovice, \\ Czech Republic \\ ${ }^{b}$ Prácheň Museum in Písek, Velké náměsti 114, 39724 Písek, Czech Republic \\ ${ }^{c}$ University of South Bohemia, Faculty of Philosophy, Institute of Archaeology, Branišovská 31 a, 37005 České Budejovice, Czech Republic \\ ${ }^{d}$ Charles University in Prague, Faculty of Arts, Institute of Prehistory and Early History, Celetná 20, 11636 Praha 1, Czech Republic \\ ${ }^{e}$ University of West Bohemia, Faculty of Philosophy and Arts, Sedláčkova 15, 30614 Plzeñ, Czech Republic \\ fUniversity of South Bohemia, Faculty of Science, Department of Parasitology, Branišovská 31, 37005 České Budějovice, Czech Republic
}

\section{ARTICLE INFO}

\section{Article history:}

Received: $28^{\text {th }}$ November 2014

Accepted: $16^{\text {th }}$ August 2015

\section{Keywords:}

Middle Ages

Central Europe

well backfill

bioarchaeology

artefact analyses

\begin{abstract}
A B S TRACT
Two secondary backfilled wells and remnants of a walled building, likely a school, were excavated during a salvage excavation of a medieval part of Písek, Bakaláře Square (South Bohemia, Czech Republic) in 2008. Well 1 was completely uncovered, whereas only the upper part of well 2 was excavated; the wells being dated to the $14^{\text {th }}$ century A.D

Well 1 was examined by bioarchaeological methods (analyses of plant macroremains, anthracology and xylotomy, pollen, dendrochronology, archaeozoology, palaeoparasitology, and diatoms), as well as by traditional archaeological typology of central European artefacts (ceramics, wood, fragments of glass, slag, and daub). It was possible to detect imported materials of various origins and to reconstruct the environment of the town and town background, as well as the common practices for hunting, growing, and waste management in medieval times.
\end{abstract}

\section{Introduction}

This paper reports on the archaeological and bioarchaeological data gained from the salvage excavation of two Medieval wells in Písek, Bakaláře Square (South Bohemia, Czech Republic) in 2008 (Figure 1). A large number of artefacts, especially ceramics and wood, were recovered, and fragments of glass, slag, and daub were also found in the wells. The following bioarchaeological methods were applied: plant macrofossil analysis, anthracology, xylotomy, palynology, dendrochronology, archaeozoology, parasitology and diatom analyses.

The first written accounts of Písek date back to 1243 A.D., when King Wenceslas I reigned. The town was established in a sparsely settled landscape, in connection with gold mining activities in the River Otava (Kudrnáč 1971) and as a control

*Corresponding author. E-mail: terezasalkova@seznam.cz point for an important trading route (Fröhlich 2013). The excavated wells are located near the Church of The Nativity of the Virgin Mary which buttresses the southern edge of the historic town centre ( 379 asl) and is elevated about 20 metres above the river.

During pavement reconstruction of Bakaláře Square which surrounds the church, archaeological salvage excavations were perfomed in 2008-2009 (Figure 2). Among the prominent finds excavated there are the remains of a tumuli and five urn graves dated to the Bronze Age period, and remnants of the Medieval and Postmedieval town. The town walls, foundations of various buildings (including the Latin school), fountain, remains of wooden tubing and other features from the medieval and early modern periods were unearthed (Houfková et al. 2013). The focus of this paper will be on the description and results from the two medieval wells. Well 1 was completely excavated, whereas well 2 was only excavated in its upper part, and the medieval infill was then conserved for the future. They are currently 


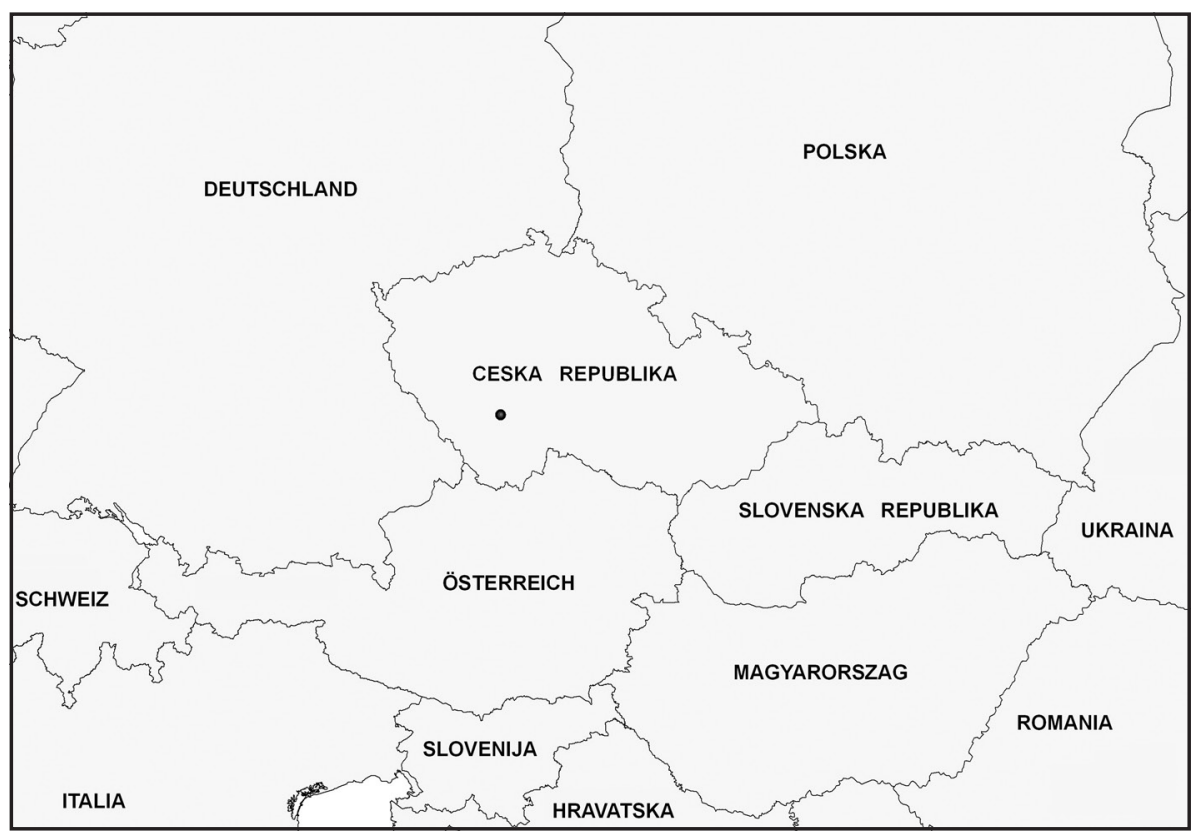

Figure 1: Bakaláře Square, Písek. Location of the site within Central Europe.

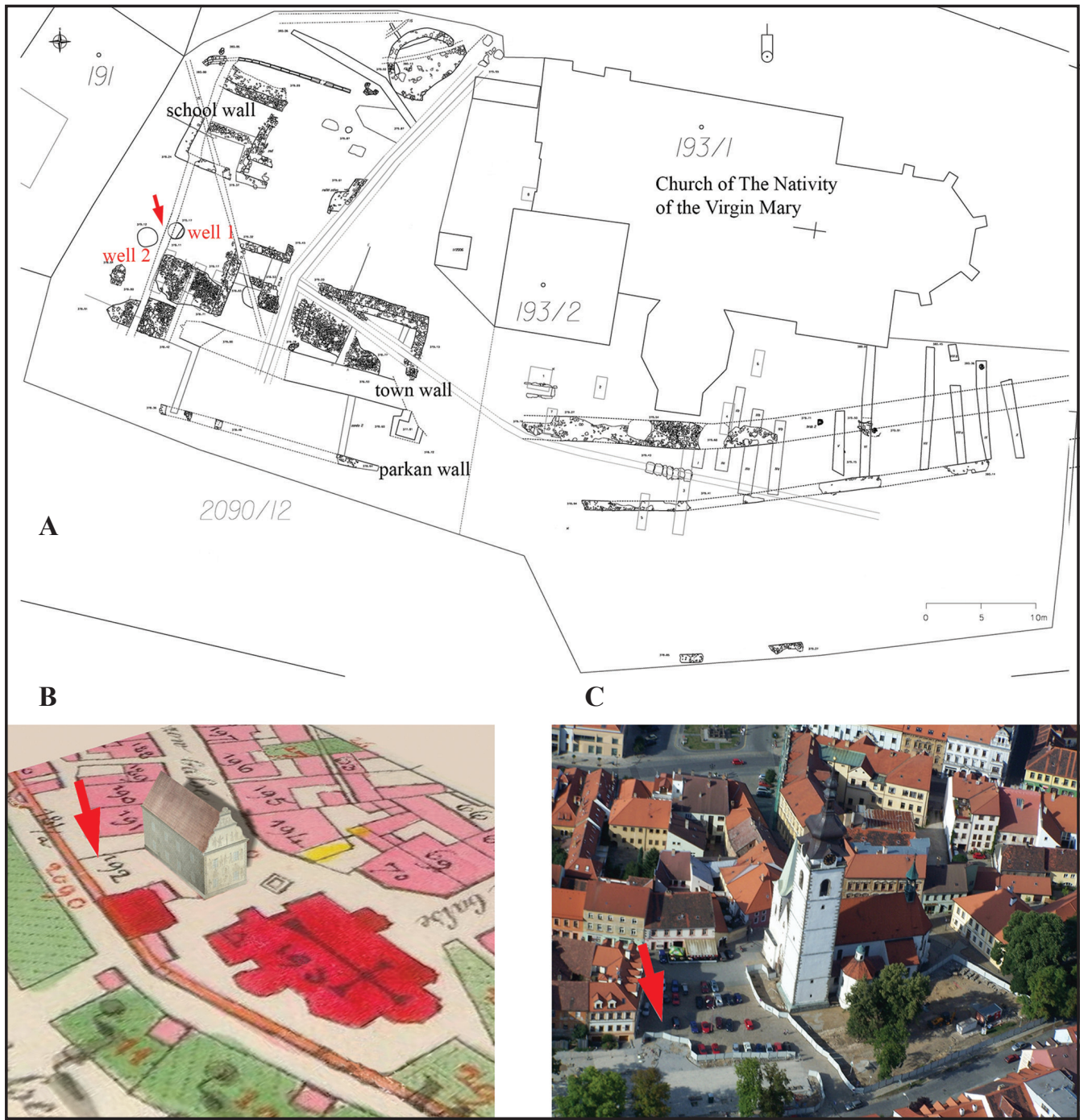

Figure 2: Bakaláře Square, Písek. A - General plan of the archaeological excavation in season 2008-09 (drawn by Geo-cz, edited by T. Šálková). B - Reconstruction of the demolished Latin School by builder Bečka in 1857 (Prácheň Museum Písek) in a map based on an Imperial imprint of the Stable cadastre of 1837 (created by Geo-cz). C - Aerial photo (photo by V. Möglich). Wells 1 and 2 are marked in red. 
located within the public space of the square, situated within the vicinity of the former Renaissance Latin school. In 2008, a foundation trench at the western front of the school was excavated. The ceramic assemblage (graphite storage vessels, pot fragments, etc.) of the trench infill could be dated to the $14^{\text {th }}$ century. A double foundation wall of the school was excavated in 1999 by Jiří Fröhlich and Eva Koppová (Fröhlich, Koppová 2000), who revealed a more complicated situation. According to Zikmund Winter, the Renaissance school was built upon the former foundations of a building that had been destroyed by fire. According to the earliest written sources the presence of the school can be dated back to at least the $15^{\text {th }}$ century (Winter 1901,117 ; for more see Hoffmann 1992, 322). The location of the school being next to the church cemetery is typical for the Middle Ages (Hoffmann 1992, 324), so it can be assumed that the wells were next to the school in medieval times. In those times, the cleaning and heating of the school buildings were usually performed by the pupils or teacher. Toilets covered by boards were usually located by the school (Hoffmann 1992, 325). Archaeological and bioarchaeological evidence from the investigation of the infill of the wells can partly contribute to solving the following important questions: For what a purpose was the well used? Which kind of material was used to fill the well and what activity and space interactions does this material reflect? The issue of primary and secondary infills of medieval wells and cesspits has been discussed for more than 50 years (see e.g. Opravil 1964; Nechvátal, Smetánka 1965; Nechvátal 1967; Široký 2000; Smith 2013). Wells were very often secondarily used as cesspits because of water contamination or changes in the water regime (e.g. for Písek, see Fröhlich 2002). Water contamination can be detected by methods of environmental archaeology (e.g. Figueiral, Séjalon 2014). We consider the feature as a well based on the presence of a dirt separator (Figure 4A, layer 3), which was at the bottom of well 1 (Houfková et al. 2013).

\section{Water management in medieval Písek}

Several excavations of medieval wells have been undertaken in the medieval part of the town of Písek (Figure 3). The well at house numbers 118 and 119 was situated near the former royal castle of Písek (Figure 3, n.3). The depth of this well was 17.2 metres, and the infill of the sludge area at the bottom of the well contained ceramic shards and a

Figure 3: Map of the town of Písek showing medieval and postmedievals wells that have been researched. The Bakaláře square site is marked by a red arrow. Map by Soukup 1910, edited by J. Jiřík and M. Pták. 1: well 1, Bakaláře; 2: well, Bakaláře; 3: well at house numbers (Nos.) 118 and 119 situated near the castle; 4: Jungmannova Street, No. 23; 5: Soukenická Street, No. 59; 6: Soukenická Street, No. 63; 7: Nerudova Street, No. 66; 8: former Dominican monastery; 9: southeast part of the large square, in front of No. 175; 10: southeast corner of Alšovo Square, in front of No. 50; 11: Havlíčkovo Square, No. 94; 12: Hejdukova Street, No. 96 (by Fröhlich, Koppová 1995, 3-19; Fröhlich 1997, 142; Adámek, Fröhlich 2002, 39; Sedláček 1912, 384, 319; Jiř́k, Pták 2010).

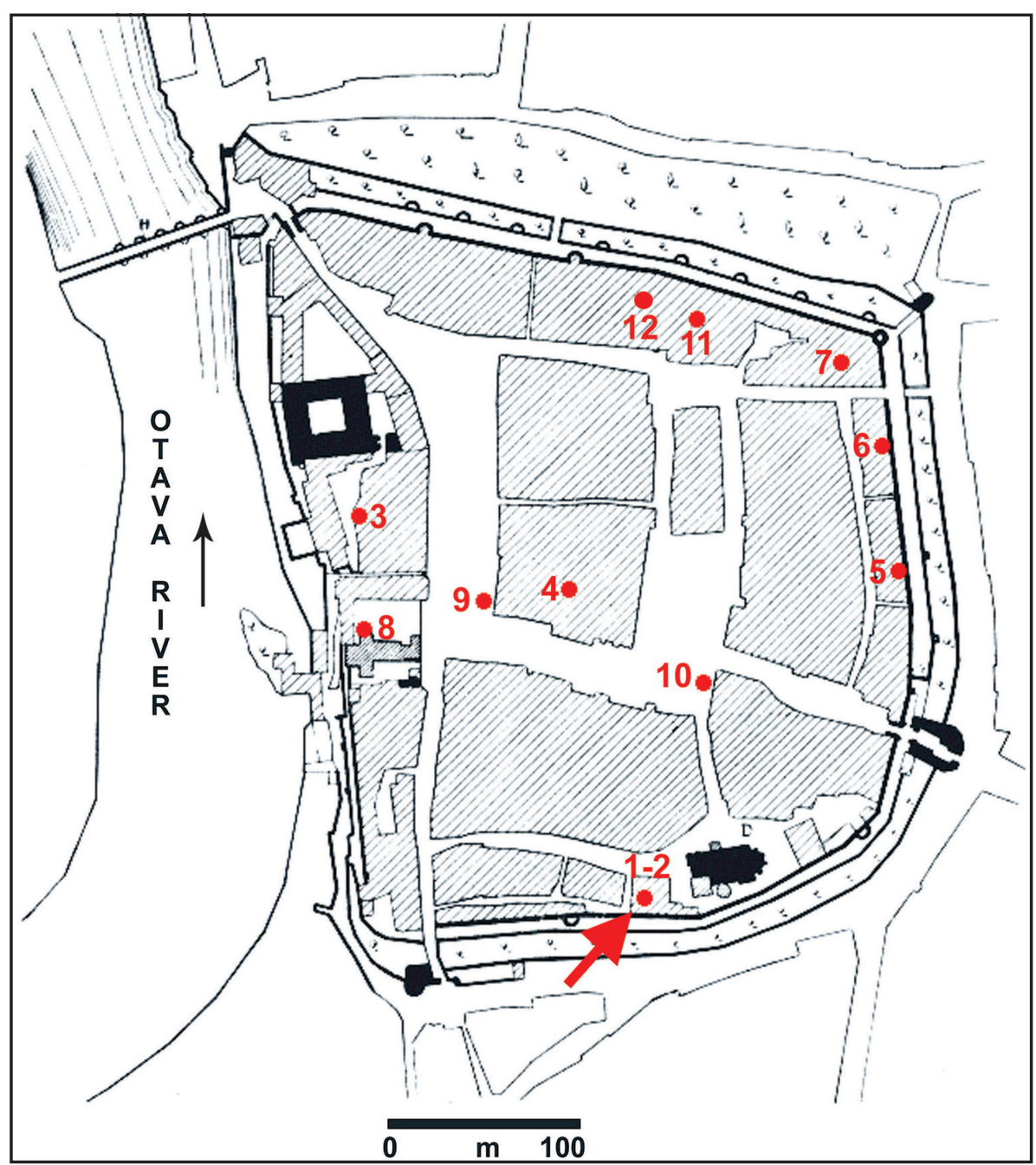


pail dating back to the $15^{\text {th }}$ century. The following layers of the well were formed of a secondary infill dated to the Late Medieval and Early Modern Age, mainly to the $16^{\text {th }}$ and $17^{\text {th }}$ centuries, when the well was used for waste disposal. The anaerobic and moist environment of the well had enabled the preservation of organic materials, such as various wooden and leather artefacts, animal bones (sheep, cow, pig, dog, and poultry), a pinecone, and a walnut fragment. Other medieval (or early modern) wells were excavated at Jungmannova Street, house number 23 (Figure 3, n. 4); Soukenická Street, house numbers 59 (Figure 3, n. 5); and 63 (Figure 3, n. 6); and Nerudova Street, house number 66 (Figure 3, n. 7). However, bioarchaeological analyses of these infills have not been undertaken (Fröhlich, Koppová 1995, 3-19; Fröhlich 1997, 142). Another well of an unclear age has been reported within the area of a contemporary courthouse, i.e. an area that was originally close or even part of the former Dominican monastery (Adámek, Fröhlich 2002, 39; Figure 3, n. 8). According to August Sedláček, there were also two municipal wells; the first was located in the southeast part of the large square (in front of house number 175; Figure 3, n. 9) and the southeast corner of Alšovo Square (in front of house number 50; (Figure 3, n. 10). The depth of the second one was estimated at 14.45 metres and was still being used in 1830 (Sedláček 1912, 384, 319; Fröhlich, Koppová 1995, 7).

Another well has been detected attached to house number 94 at Havlíčkovo Square. The total depth of 8-9 metres was estimated by Jindřich Kurz in 1996 (Jiřík, Pták 2010; Figure 3, n. 11). The last specimen was recorded in Hejdukova Street, house number 96 (Fröhlich, Koppová 1995, 7), but this well has not been excavated and its dating is unknown (Figure 3, n. 12).

\section{Material and methods}

The $7.70 \mathrm{~m}$ deep well 1 (Figure 4A) has been carved into the gneiss (Figure 4B). At nearly $4 \mathrm{~m}$ thick, layer 1 consisted of fragments of daub often carrying construction imprints and large iron slags. Layer 2, which was black and muddy, was deposited between 4 and 7.6 metres. In the bottom (Figure 4C), layer 3 situated in the dirt separator was dark, muddy and sandy. An important question is the composition of layer 3 and its connection with the loss of the primary function of the wells (e.g. polluted water). Well 2, layer 1 was excavated in its upper part, the character of this layer being analogical to well 1, layer 1 .

\subsection{Field work, well infill sampling}

The well 1 infill (Figure 4A) was stratigraphically excavated by a pulley system with an electric drive. Ceramic fragments, archaeozoological material, and fragments of

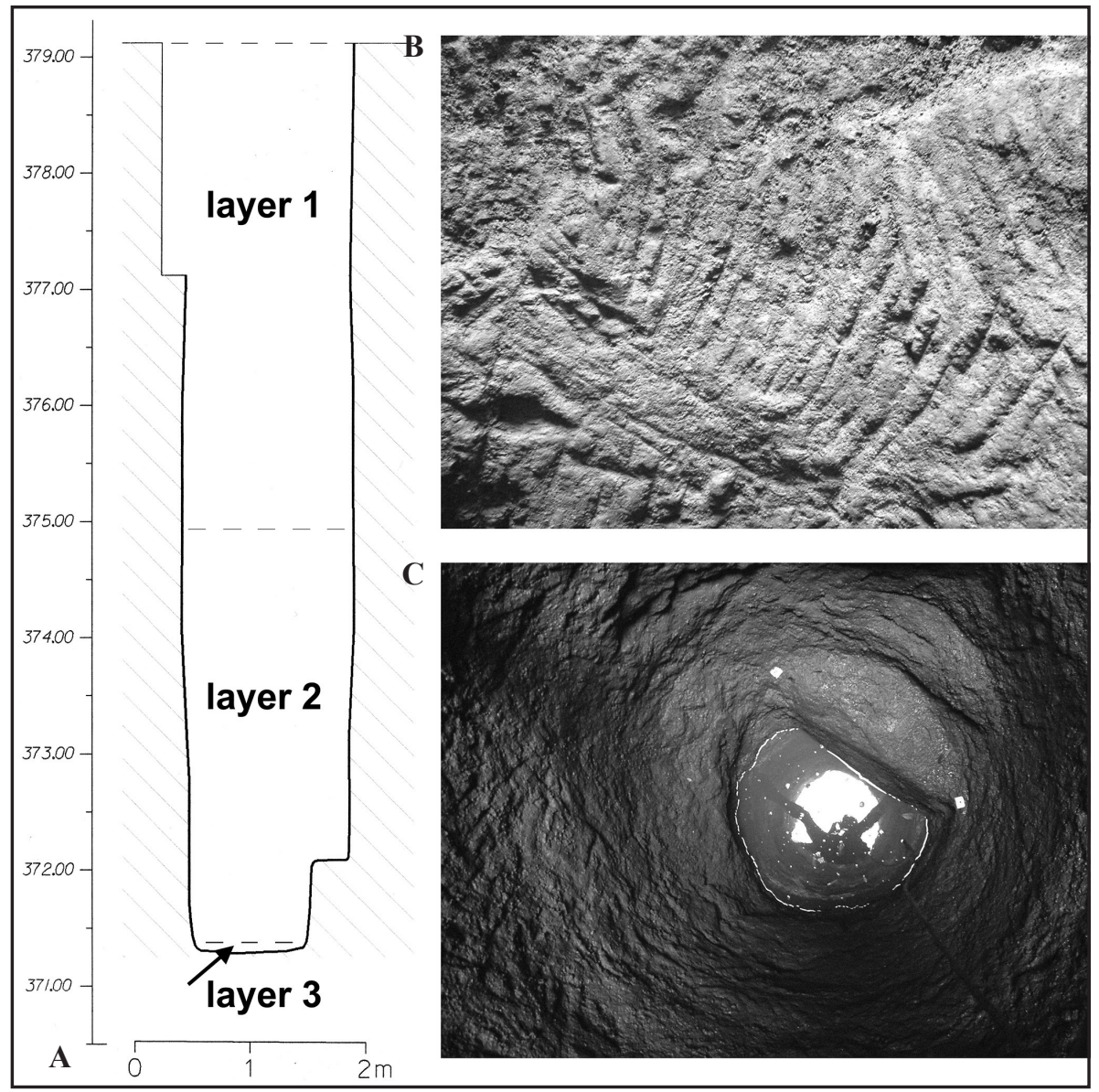

Figure 4: Bakaláře Square, Písek, well 1. A - cross-section of well 1 (plan by GEOcz). B - detail of tool traces. C - bottom of the well with detail of the soil filter (photo by B. Vácha). 
wooden artefacts were taken out during the excavation and during sieving of all deposits. Sediments were separated with a $1 \mathrm{~cm}$ mesh sieve, and one of the wooden fragments was dendrodated. Samples of sediments were taken before sieving for artefact separation and charcoal and wood remains were separated by water flotation $(0.2 \mathrm{~mm}$ mesh). Sample 1 was taken out of layer 2 (volume 20 litres), and sample 2 was taken out of layer 3 at the bottom of the well (volume 8 litres). Flotation residue (1.8 $\mathrm{mm}$ mesh) was also analysed, and small fragments of glass were found. The sample of sediment from layer 3 was subsampled, chemically processed, and analysed for pollen, parasites, and diatoms.

Well 2, layer 1 was excavated only in its upper part. Artefacts and animal bones were analysed. Bioarchaeological analyses were not applied.

\subsection{Dating methods and artefact types}

The well infill material could be dated using dendrochronology and ceramic typology. Many fragments of wooden artefacts were found in layer two. Two samples identified as wood of fir and spruce were used for dendrochronological dating; tree-ring widths were measured at an accuracy of $0.01 \mathrm{~mm}$ using a measuring device (TimeTable) and Past 4 software (Knibbe 2004) and the master chronologies of fir and spruce for the Czech Republic (made by T. Kyncl).

During the excavation of both wells (upper part of well 2 and in all three layers of well 1), a number of pottery fragments were obtained, which were used as one of the pillars for dating the abandonment of the well. Some of the finds were collected during the excavation; the rest were obtained during sieving (Figures 5, 6; Reichertová 1965; Richter, Krajíc 2001; Procházka 1994; Nekuda, Reichertová 1968; Radoměrský, Richter 1976; Vařeka 1998; Orna ed. 2011).

Many daub fragments with imprints of construction and iron slag fragments were found in well 1, layer 1 (Figure 4) and well 2, layer 1. One fragment of iron slag from well 1 was assessed with X-Ray Fluorescent Analysis. Wooden fragments of joists, boards, planks, chips, twigs, and birch and pine bark were found in well 1 , layer 3 . Many tiny fragments of opalised glass were identified in flotation residues of well 1, layer 3 .

\subsection{Bioarchaeological methods}

Bioarchaeological methods were applied to interpret the sediment character. The following finds were analysed: plant macroremains, charcoal and wood, pollen, diatoms, paleoparasites and bones. Botanical taxa were categorised into groups based on the international ArboDat Multi database (Kreuz, Schäfer 2002; Pokorná et al. 2011).

Plant macroremains were picked out and microscopically (Olympus SZ51, Nicon SZM 1500) determined according to Berggren (1981); Anderberg (1994); Cappers et al. (2006; 2009); and the comparative seed collection from the Laboratory of Archaeobotany and Palaeoecology, Faculty of Science, University of South Bohemia. Carbonised, waterlogged, and mineralised remains were quantified. The plant species were ordered into general eco-groups according to the specific and environmental requirements of each species (Hejný, Slavík eds. 1988-1992; Slavík ed. 19952000; Slavík, Štěpánková eds. 2004; Štěpánková ed. 2010). Percentage ratios of eco-groups based on the abundance of particular subfossil and charred macroremains were plotted, along with the percentage ratios of eco-groups of pollen types using Tilia 1.5.12. software (Grimm 2011; Figure 7).

Charcoal and wood analysis was performed on 200 fragments from the largest fraction $(>2 \mathrm{~mm}$ ) per sample. The charcoals were identified using an episcopic interference microscope (Nikon Eclipse 80i) with 200-500× magnification and the comparative reference collection; additional standard identification keys were also used (Schweingruber 1990; Heiss 2000). Species abundance recorded by wood and charcoal remains was plotted in histograms along with the percentage ratios of arboreal pollen types using Tilia 1.5.12. software (Grimm 2011; Figure 7).

A sediment sample for pollen analysis $(1 \mathrm{~g})$ was taken from layer 3 at the bottom of well 1. Extraction of pollen grains was done by chemical treatment according to Faegri and Iversen (1989). Pollen grains were counted in light microscopy (LM) at a magnification of $400-1000 \times, 481$ determinations being recorded. Taxonomic identifications followed Punt et al. (1976-2009) and Beug (2004). Relative abundance of pollen types was presented as percentages of the total pollen sum (TPS). Non-pollen palynomorphs (NPPs) were counted along with the pollen, and were calculated as percentages of the TPS+NPP sum. Half of the sample volume prepared for the pollen analysis (Faegri, Iversen 1989) was used for further parasitological investigation. These results were compared with the results originating from the other preparation method; the sample originating from the same layer 3 in the bottom of well 1 was rehydrated in a solution of $0.5 \%$ trisodium phosphate (Callen, Cameron 1960) and treated with two techniques: sedimentation AMS III concentration technique and flotation - sheather sugar solution (500 g sucrose, $6.5 \mathrm{~g}$ phenol, $320 \mathrm{ml}$ distilled water). One sample from the bottom (layer 3 ) of well 1 was analysed for diatoms. Organic matter from sediment samples was digested in hydrogen peroxide following standard procedures (Battarbee 1986). Permanent slides were prepared using Pleurax (Fott 1954) as a mounting medium. The samples were studied with a LM (Olympus BX 51) at a magnification of $1000 \times$. Diatom analyses were processed but with a negative result.

Bones from wells 1 and 2 were analysed. Faunal spectrum was established by using the NISP (Number of Identified Specimens) and MNI (Minimum Number of Individuals) calculated from bone and dental remains. The age at death for pigs was estimated from the abrasion stages of the lower jaw teeth (Grant 1982); for sheep and goat we used the method developed by Helmer (1995; see Helmer, Vigne 2004). In some cases, the degree of epiphyseal fusion and closure of cranial sutures in animals were registered (Silver 1969). The distinction between sheep and goats depended on morphological features (Prummel, Frisch, 1986) and 

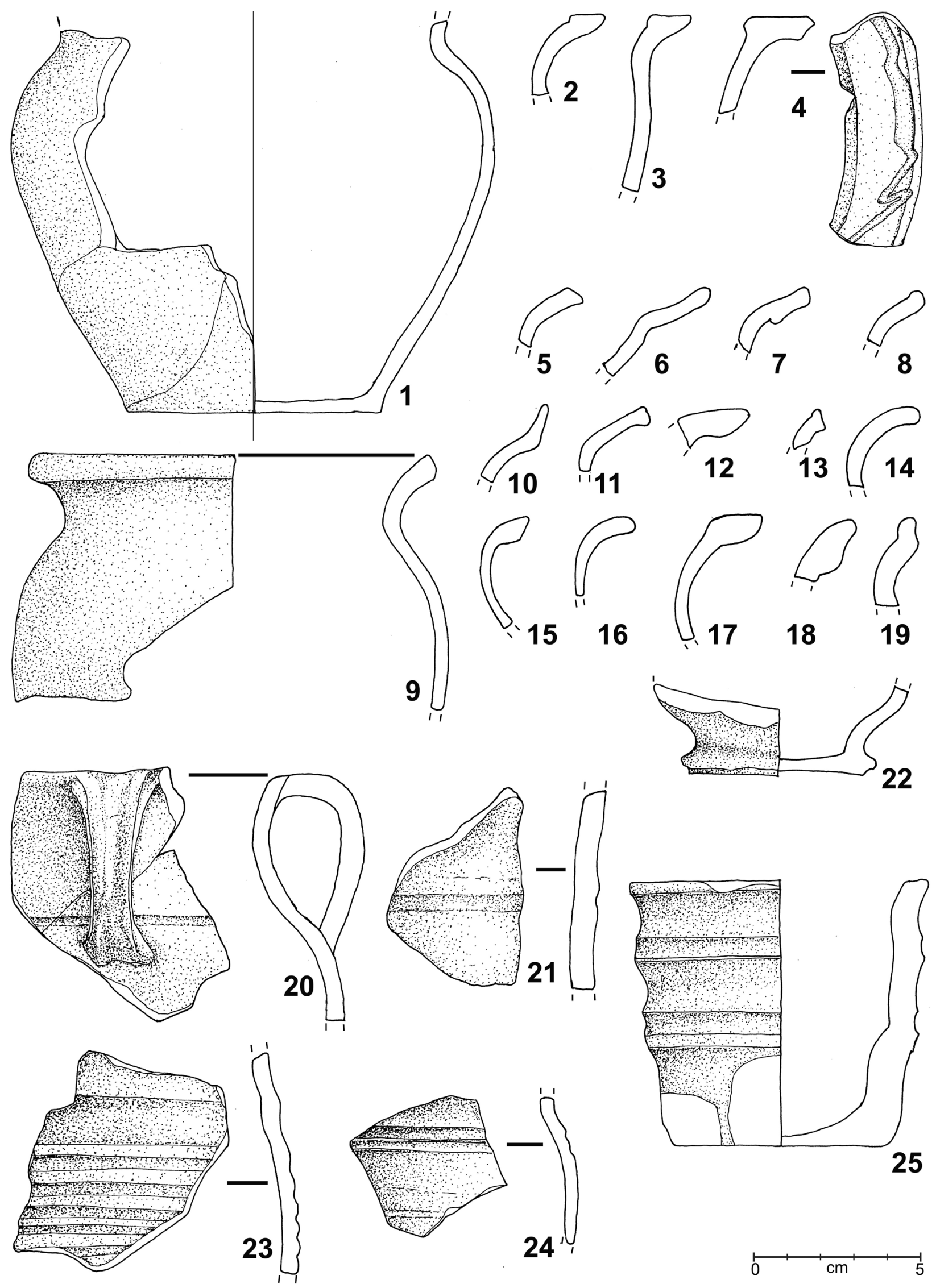

Figure 5: Bakaláře Square, Písek, well 1. Fragments of kitchen pottery. Drawing by T. Šálková, edited by M. Pták. 

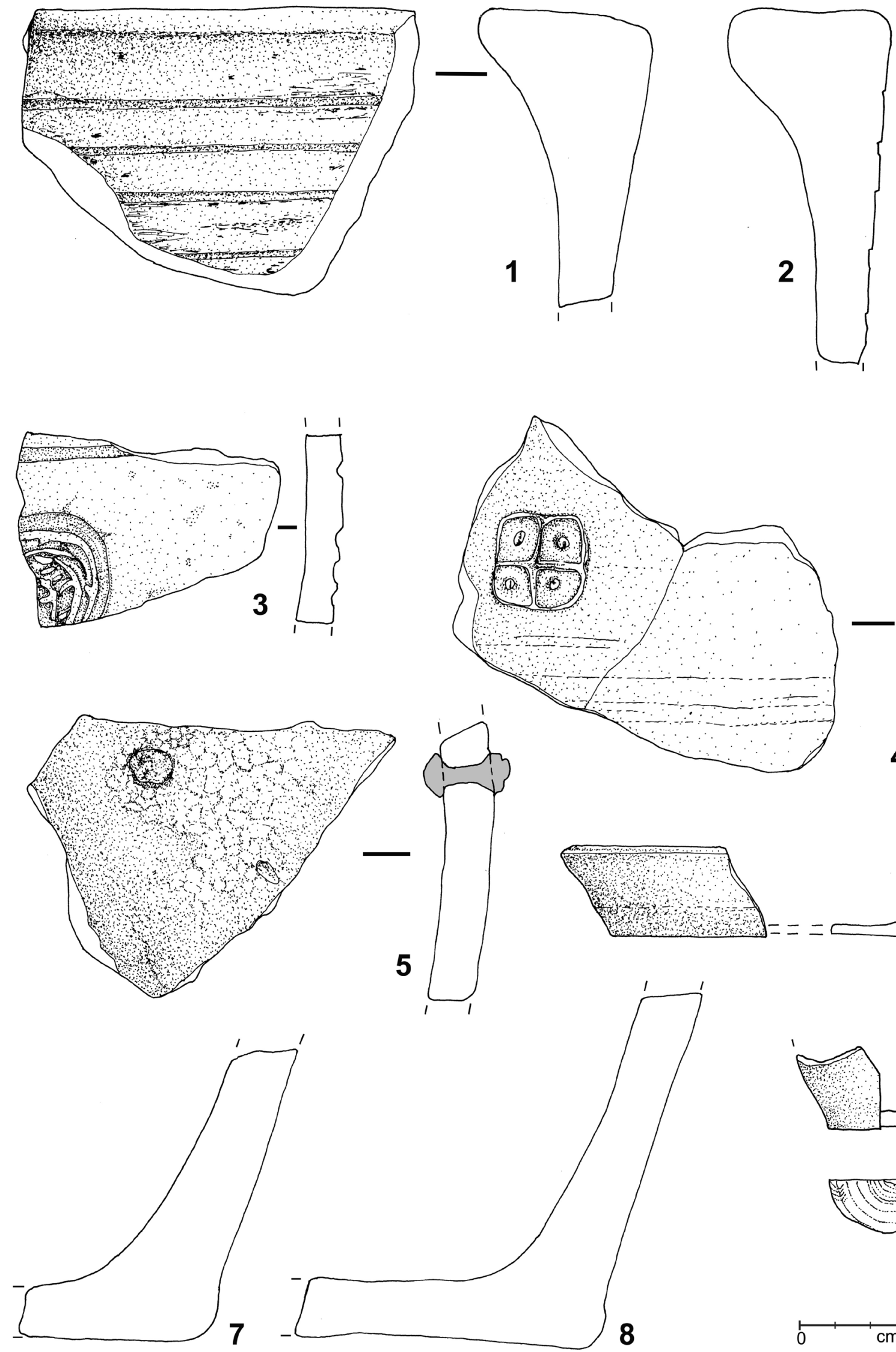

4
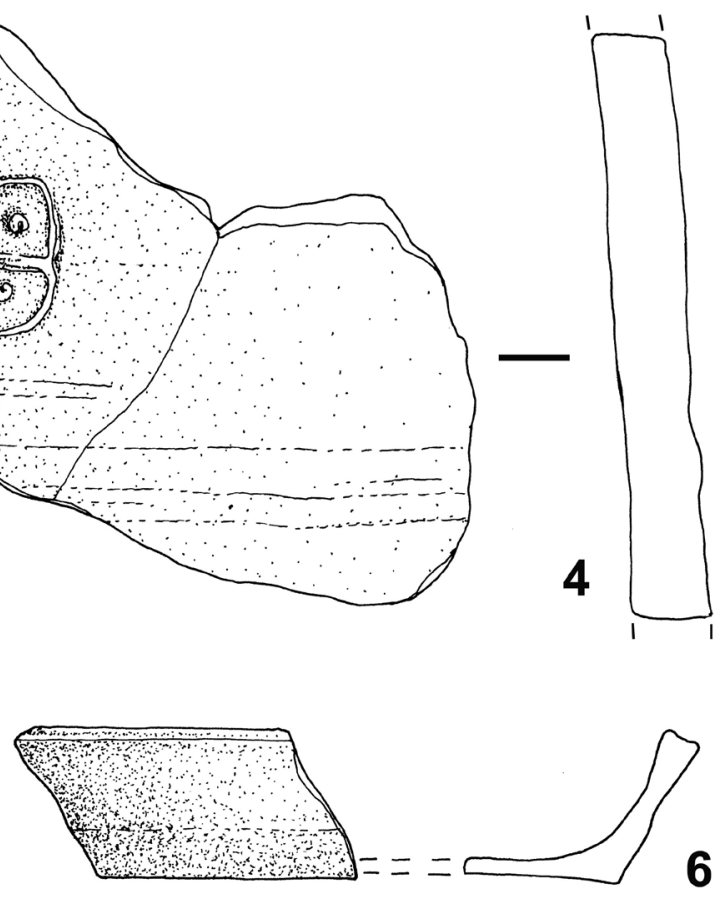

6

9
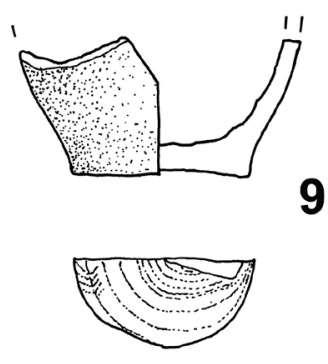

8

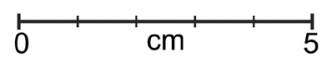

Figure 6: Bakaláre Square, Písek, well 1. Fragments of storage and imported pottery. Drawing by T. Š́lková, edited by M. Pták. 


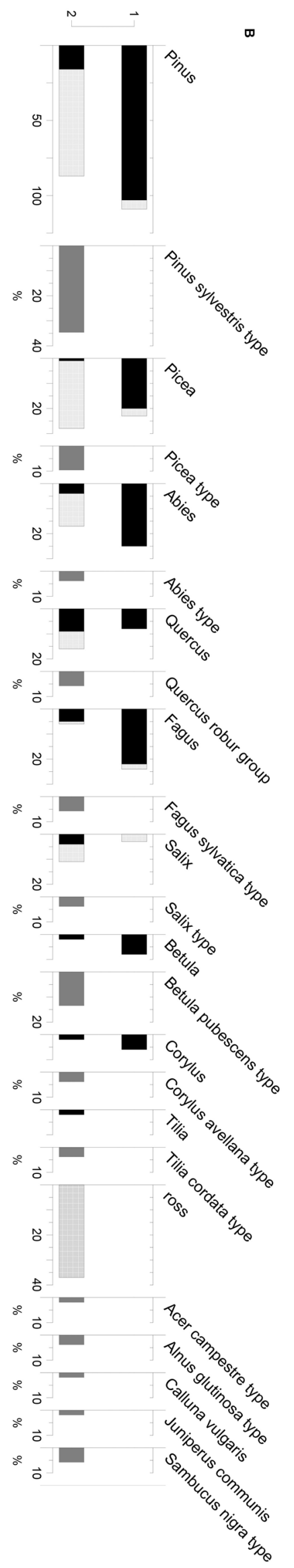

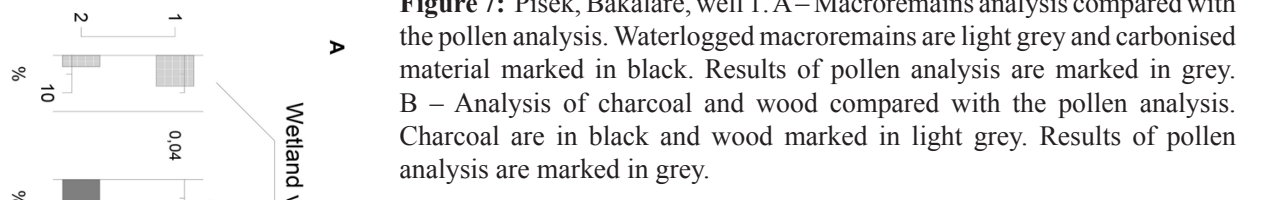

additional information from an assemblage's history (e.g. fragmentation during butchering or weathering) guided the taphonomic analysis.

\section{Results}

\subsection{Wooden artefacts and dendrochronology}

Hundreds of wooden fragments were recovered from the sediment of well 1, layer 2. Most of the fragments are considered as being from production and construction waste. Among the tools, a wooden whisk was found. Only one piece of fir wood (Abies) could be dated by dendrochronology to the year 1318 A.D., but the sample did not include the outermost ring. Being a relatively small piece of wood, an unknown amount of tree rings could have been removed during the processing, but the date does give some idea concerning the start of the well's infilling process.

\subsection{Ceramics}

Kitchen and storage pottery and fragments of bricks, tiles, and pantiles (roof tile) were found. Almost 500 fragments of ceramic vessels were obtained from well 1 . Only the top layer of well 2 was excavated. Ceramic fragments from both wells can be dated to the $14^{\text {th }}$ century (see discussion). Kitchen pottery was represented by fragments of pots (e.g. Figure 5: 1, 9, 23), pitchers (Figure 5: 20), and bowls/lids (e.g. Figure 5: 6, 22). One fragment of a burner or low bowl (Figure 5: 6), and two fragments of a cup or bowl (Figure 5: 25) were also collected. The pottery rim profiling varies (e.g. Figure 5), ledge and horizontally everted rims being dominant. Horizontally-grooved rims were present, and some vessel bottoms were marked by pouring sand underneath. Horizontal engraved decoration was prominent, and decoration by tracing wheels was not represented in the ceramic collection; reduction firing was found to be dominant.

A torso of a thin-walled vessel made of light clay (Figure 6:9) is unique; it was made on a potter's wheel, with the underside carrying string cut traces.

Many fragments of storage pottery with a mixture of graphite were found. The vessels are characterised by large inverted edges (Figure 6: 1,2), and the decoration consisted of both single and multiple horizontal grooves. Some graphite vessels were marked by stamps (Figure 6:3,4), and repair holes were also frequent (Figure 6: 5).

\subsection{Other artefacts}

Many fragments of iron slags were found in layer 1; X-Ray Fluorescent Analysis revealed that one sample contained 
Table 1. Bakaláŕe Square, Písek, well 1. List of plant macroremains (c - carbonised; $n$ - waterlogged), wood (w) and charcoal (ch), in NISP (Number of Identified Specimens).

\begin{tabular}{|c|c|c|c|c|c|c|c|c|c|c|c|}
\hline Sample & & 1 & 2 & Sample & & 1 & 2 & Sample & & 1 & 2 \\
\hline Abies alba & $\mathbf{n}$ & & 2 & Hordeum vulgare var. vulgare $\mathrm{r}$ & c & & 6 & Ranunculus cf. repens & n & 6 & 1 \\
\hline Abies & ch & 25 & 4 & Hordeum vulgare var. vulgare & $\mathbf{n}$ & & 3 & Ranunculus flammula & $\mathbf{n}$ & 82 & \\
\hline Abies & $\mathbf{w}$ & & 13 & cf. Hordeum vulgare & c & 2 & & Ranunculus sp. & n & 5 & 3 \\
\hline Agrostemma githago & $\mathbf{n}$ & 4 & 2 & Humulus lupulus & n & 37 & 40 & cf. Rosaceae & n & & 2 \\
\hline Ajuga genevensis & $\mathbf{n}$ & & 1 & Hyoscyamus niger & $\mathbf{n}$ & 3 & & Rubus fruticosus & c & 2 & \\
\hline Alchemilla/Afarens & $\mathbf{n}$ & 2 & & Hypericum perforatum & n & 56 & 5 & Rubus fruticosus & n & 3 & 2 \\
\hline Anagalis arvensis & $\mathbf{n}$ & 9 & 2 & Chenopodium album & $\mathbf{n}$ & 774 & 138 & cf. Rubus fruticosus & c & 2 & 6 \\
\hline Anthemis arvensis & $\mathbf{n}$ & 5 & 2 & Chenopodium ficifolium & n & 53 & 12 & Rubus ideaus & c & & 1 \\
\hline Apiaceae & $\mathbf{n}$ & 2 & 1 & Chenopodium hybridum & $\mathbf{n}$ & 7 & 1 & Rubus ideaus & $\mathbf{n}$ & 14 & 5 \\
\hline Arenaria serpyllifolia & $\mathbf{n}$ & 65 & 1 & Chenopodium sp. & n & 32 & & Rumex acetosella & n & 78 & 6 \\
\hline Asteraceae & $\mathbf{n}$ & 5 & 3 & cf. Juniperus communis & $\mathbf{n}$ & & 1 & Rumex obttusifolius/crispus & $\mathbf{n}$ & 6 & \\
\hline Atriplex sp. & $\mathbf{n}$ & 33 & 2 & Lamiaceae & $\mathbf{n}$ & 1 & 1 & Rumex sp. & $\mathbf{n}$ & & 1 \\
\hline Betula & ch & 8 & 2 & Lamium cf. amplexicaule & $\mathbf{n}$ & 9 & & Salix & ch & & 4 \\
\hline Cannabis sativa & $\mathbf{n}$ & 2 & & Lamium sp. & $\mathbf{n}$ & & 1 & Salix & $\mathbf{w}$ & 3 & 7 \\
\hline Carex cf. brizoides & $\mathbf{n}$ & 6 & & Lapsana communis & $\mathbf{n}$ & 15 & 18 & Scirpus sylvaticus & n & 70 & 1 \\
\hline Carex cf. contigua & $\mathbf{n}$ & 1 & & cf. Leucosinapis alba & c & & 1 & Scleranthus annuus & $\mathbf{n}$ & 12 & 4 \\
\hline Carex cf. disticha & $\mathbf{n}$ & 84 & 2 & cf. Lolium perenne & $\mathbf{n}$ & 1 & & Secale cereale & c & 27 & 1 \\
\hline Carex cf. hirta & $\mathbf{n}$ & 5 & 1 & Lycopus europaneus & $\mathbf{n}$ & 2 & & cf. Secale cereale & c & 2 & \\
\hline Carex cf. pallescens & $\mathbf{n}$ & 27 & & Lychnis floss cuculi & $\mathbf{n}$ & 3 & & Setaria cf. viridis & $\mathbf{n}$ & & 1 \\
\hline Carex cf. vulpina & $\mathbf{n}$ & 12 & & Malus/Pyrus & $\mathbf{n}$ & 1 & 1 & Silene vulgare & $\mathbf{n}$ & 25 & 9 \\
\hline Carex sp. & $\mathbf{n}$ & 4 & & Mentha cf. aquatica & $\mathbf{n}$ & 1 & 1 & Solanum dulcamara & $\mathbf{n}$ & 6 & 7 \\
\hline Centaurea cyanus & $\mathbf{n}$ & 7 & 6 & Mentha cf. aquatica & $\mathbf{m}$ & 1 & & Sonchus cf. oleraceus & $\mathbf{n}$ & & 1 \\
\hline Cerealia & c & 42 & 7 & Neslia paniculata & c & 1 & & Stachys arvensis & n & 1 & \\
\hline Cerealia straw & $\mathbf{n}$ & 4 & 2 & Neslia paniculata & $\mathbf{n}$ & 3 & 5 & Stellaria graminea & c & & 1 \\
\hline Cirsium cf. arvense & $\mathbf{n}$ & & 5 & Panicum miliaceum & c & 1 & & Stellaria graminea & $\mathbf{n}$ & 19 & 8 \\
\hline Clinopodium vulgare & $\mathbf{n}$ & 1 & & Panicum miliaceum & n & 1 & 36 & Stellaria media & n & 121 & 10 \\
\hline Corylus avellana frag. & $\mathbf{n}$ & 13 & 8 & Papaver rhoeas & $\mathbf{n}$ & 12 & & Thlaspi arvense & $\mathbf{n}$ & 14 & 8 \\
\hline Corylus & ch & 6 & 2 & Persicaria lapatifolia & $\mathbf{n}$ & 13 & 5 & Tilia & ch & & 2 \\
\hline Crateagus sp. & $\mathbf{n}$ & & 1 & Picea & ch & 20 & 1 & Trifolium arvense & $\mathbf{n}$ & 2 & \\
\hline Cyperaceae & $\mathbf{n}$ & 8 & 3 & Picea & $\mathbf{w}$ & 3 & 27 & Triticum aestivum & c & 1 & \\
\hline cf. Daucus carota & $\mathbf{n}$ & 1 & 3 & Pinus & c & & 1 & $\begin{array}{l}\text { Triticum aestivum/ } \\
\text { compactum }\end{array}$ & c & 6 & \\
\hline Dianthus deltoides & $\mathbf{n}$ & 3 & & Pinus & $\mathbf{n}$ & 4 & 4 & Urtica dioica & n & 57 & 5 \\
\hline Eleocharis sp. & $\mathbf{n}$ & 31 & 2 & Pinus & ch & 103 & 16 & Urtica urens & n & 5 & 1 \\
\hline Fagus & $\mathbf{w}$ & 2 & 1 & Pinus & $\mathbf{w}$ & 6 & 71 & Valerianella dentata & $\mathbf{n}$ & 43 & 8 \\
\hline Fagus & ch & 22 & 5 & Poaceae & n & 4 & & Verbena officinalis & $\mathbf{n}$ & 3 & \\
\hline Fallopia convolvulus & $\mathbf{n}$ & 37 & 14 & Poaceae leaf & n & 3 & 2 & Viola sp. & c & 1 & \\
\hline Ficus carica & $\mathbf{n}$ & 18 & 32 & Polygonum aviculare & $\mathbf{n}$ & 219 & 33 & Viola sp. & n & 44 & 6 \\
\hline Fragaria sp. & $\mathbf{n}$ & 35 & & cf. Populus tremula & n & 15 & & Vitis vinifera & c & & 1 \\
\hline Fragaria vesca & $\mathbf{n}$ & 22 & 7 & Potentila erecta & n & 19 & & Vitis vinifera & n & 1 & \\
\hline Galeopsis tetrahit/bifida & $\mathbf{n}$ & & 2 & Prunella vulgaris & n & 4 & 3 & indeterminata & n & 17 & 3 \\
\hline Galium palustre & c & 1 & & Prunus sp. & $\mathbf{n}$ & 1 & 2 & bark & n & & 37 \\
\hline Galium spurium & c & 2 & & Prunus spinosa & $\mathbf{n}$ & 2 & & bud & c & & 2 \\
\hline Galium spurium & $\mathbf{n}$ & 6 & & Quercus & ch & 8 & 9 & bud & n & 43 & 14 \\
\hline Geranium cf.dissectum & $\mathbf{z}$ & 1 & & Quercus & $\mathbf{w}$ & & 7 & leaf & $\mathbf{n}$ & 2 & 2 \\
\hline Geranium disectum & $\mathbf{n}$ & 36 & 10 & cf. Quercus & $\mathbf{n}$ & 43 & 11 & moss & n & 5 & 8 \\
\hline Hordeum vulgare & c & 6 & & cf. Thalictrum flavum & n & & 1 & & & & \\
\hline
\end{tabular}

iron, barium, lanthanum, and cerium. As previously mentioned, many small fragments of opalised glass were found in layer three, but it was impossible to determine the type of glass artefact.

\subsection{Plant macroremains}

Two samples (sample 1: layer 2, 20 litres; sample 2: layer 3, 8 litres; Figure 4A) were analysed for plant macroremains: 3,193 determinations and 99 taxa were recorded (wood and 
Table 2. Bakaláře Square, Písek, well 1. Waterlogged macroremains of well 1 in Bakaláře Square, Písek; A: layer two, sample one; B: layer three, sample two. Macroremains were categorized into groups and ranked by frequency in the sample.

\begin{tabular}{|c|c|c|}
\hline \multirow{13}{*}{ 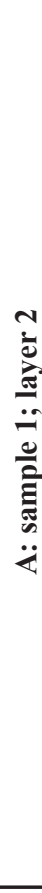 } & segetal/ruderal & $\begin{array}{l}\text { Chenopodium album, Polygonum aviculare, Stellaria media, Chenopodium ficifolium, Valerianella } \\
\text { dentata, Fallopia convolvulus, Atriplex sp., Chenopodium sp., Lapsana communis, Thlaspi arvense, } \\
\text { Papaver rhoeas, Scleranthus annuus, Anagalis arvensis, Lamium amplexicaule, Centaurea cyanus, } \\
\text { Chenopodium hybridum, Galium spurium, Neslia paniculata }\end{array}$ \\
\hline & segetal & $\begin{array}{l}\text { Rumex acetosella, Arenaria serpyllifolia, Geranium dissectum, Anthemis arvensis, Agrostemma } \\
\text { gythago, Stachys arvensis }\end{array}$ \\
\hline & ruderal/wetland & Urtica dioica, U. urens \\
\hline & segetal/ruderal/ wetland & Persicaria lapatifolia, Rumex obttusifolius/crispus \\
\hline & ruderal & Hyoscyamus niger \\
\hline & ruderal/meadows & Lolium perenne, cf. Daucus carota \\
\hline & wetland plants & $\begin{array}{l}\text { Carex cf. disticha, Ranunculus flammula, Scirpus sylvaticus, Humulus lupulus, Eleocharis sp., Carex } \\
\text { cf. pallescens, Potentila erecta, Stellaria graminea, Carex vulpina, Cyperaceae, Carex cf. brizoides, } \\
\text { Ranunculus repens, Solanum dulcamara, Carex hirta, Ranunculus sp., Lycopus, Carex cf. contigua, } \\
\text { Galium palustre, Mentha aquatica }\end{array}$ \\
\hline & meadows & Alchemilla/Afarens, Poaceae, Prunella vulgaris \\
\hline & dry environment & $\begin{array}{l}\text { Hypericum perforatum, Silene vulgare, Dianthus deltoides, Verbena officinalis, Trifolium arvense, } \\
\text { Clinopodium vulgare }\end{array}$ \\
\hline & buds and needles & indeterminata buds, cf. Quercus, cf. Populus tremula, Pinus sp. \\
\hline & other useful plants & $\begin{array}{l}\text { Fragaria sp., Fragaria vesca, Rubus ideaus, Corylus avellana, Rubus fruticosus, Cannabis sativa, } \\
\text { Prunus spinosa, Prunus spinosa, Prunus sp., Vitis vinifera }\end{array}$ \\
\hline & imported fruit & Ficus carica \\
\hline & cereals & Cerealia, Panicum miliaceum \\
\hline \multirow{13}{*}{ 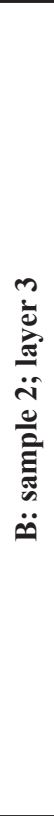 } & segetal/ruderal & $\begin{array}{l}\text { Chenopodium album, Polygonum aviculare, Lapsana communis, Fallopia convolvulus, Chenopodium } \\
\text { ficifolium, Stellaria media, Valerianella dentata, Thlaspi arvense, Centaurea cyanus, Neslia paniculata, } \\
\text { Cirsium cf. arvense, Scleranthus annuus, Atriplex sp., Anagalis arvensis, Chenopodium hybridum, } \\
\text { Sonchus oleraceus }\end{array}$ \\
\hline & segetal & $\begin{array}{l}\text { Geranium dissectum, Rumex acetosella, Agrostemma gythago, Anthemis arvensis, Arenaria } \\
\text { serpyllifolia, Setaria viridis }\end{array}$ \\
\hline & ruderal/wetland & Urtica dioica, U. urens \\
\hline & ruderal & Galeopsis tetrahit/bifida \\
\hline & ruderal/meadows & cf. Daucus carota \\
\hline & segetal/ruderal/wetland & Persicaria lapatifolia \\
\hline & wetland plants & $\begin{array}{l}\text { Humulus lupulus, Stellaria graminea, Solanum dulcamara, Cyperaceae, Ranunculus sp., Carex cf. } \\
\text { disticha, Eleocharis sp., Scirpus sylvaticus, Ranunculus repens, Carex hirta, Mentha aquatica, cf. } \\
\text { Thalictrum flavum, Rumex sp. }\end{array}$ \\
\hline & meadows & $\begin{array}{l}\text { Geranium dissectum, Rumex acetosella, Agrostemma gythago, Anthemis arvensis, Arenaria } \\
\text { serpyllifolia, Setaria viridis }\end{array}$ \\
\hline & dry environment & Silene vulgare, Hypericum perforatum \\
\hline & buds and needles & indeterminata buds, cf. Quercus sp., Pinus sp., Abies alba, cf. Juniperus communis \\
\hline & other useful plants & Corylus avellana, Fragaria vesca, Rubus ideaus, Rubus fruticosus, Prunus sp. Malus/Pyrus, Crateagus sp. \\
\hline & imported fruit & Ficus carica \\
\hline & cereals & Panicum miliaceum, Hordeum vulgare, Cerealia \\
\hline
\end{tabular}

charcoal are excluded; Table 1). The concentrations of the plant macroremains was 130.45 per litre in sample 1 and 73 per litre in sample 2. Both samples consisted of carbonised and waterlogged plant remains (waterlogged: sample 1: 96.5\%; sample 2: 95.3\%). Carbonised macroremains were represented mainly by cereals (sample 1: $91 \%$; sample 2: $51 \%)$.

\subsubsection{Carbonised macroremains}

The predominant species in sample 1 were the following cereals: Cerealia, Secale cereale, Hordeum vulgare, Triticum aestivum/compactum, Panicum miliaceum; useful fruit
(Rubus fruticosus); segetal (Geranium dissectum); segetal/ ruderal (Galium spurium, Neslia paniculata); and wetland plants (Galium palustre; Figure 7). Sample 2 contained a noticeable amount of cereals (Cerealia, Hordeum vulgare, Secale cereale) and useful fruit (Rubus fruticosus, Rubus ideaus, Vitis vinifera), whereas evidence of segetal (Leucosinapis alba) and wetland (Stellaria graminea) plants was rare (Table 1; Figure 7).

\subsubsection{Waterlogged macroremains}

Sample 1 in the category of waterlogged macroremains (Tables 1 and 2A, Figure 7) provided strong evidence of 
plants typical for rubble and field: $58 \%$ of segetal/ruderal, $8 \%$ of segetal, $3 \%$ of ruderal/wetland, and $1 \%$ segetal/ ruderal/wetland were recorded.

Evidence of wetland plants formed $17 \%$ of the waterlogged plant macroremain evidence. Buds and needles of trees represented $4 \%$ of the waterlogged macroremains, and evidence of macroremains typical for dry environments, as well as other useful plants, formed $4 \%$. Imported fruits were marginally recorded (1\%); evidence of plant macroremains typical for meadows came at $1 \%$, and cereals were marginally represented (Figure 7).

Sample 2 in the category of waterlogged macroremains (Tables 1 and 2B, Figure 7) indicated a dominance of plants typical for ruderal sites and field: $50 \%$ segetal/ruderal, $4 \%$ segetal, $1 \% \mathrm{ruderal} / \mathrm{wetland}$, ruderal, ruderal/meadows, and segetal/ruderal/wetland plants. Evidence of wetland plants formed $13 \%$ of waterlogged plant macroremain evidence, buds of trees and needles were represented by $6 \%$ of waterlogged macroremains, and evidence of macroremains typical for dry environment was formed by $3 \%$ of determinations; other useful plants represented $8 \%$ of waterlogged macroremains, especially imported fruit (6\%), $8 \%$ of waterlogged macroremains was represented by cereals, and evidence of plant macroremains typical for meadows was 1\% (Figure 7).

\subsubsection{Cereals}

Cereals accounted for $3.6 \%$ of the macroremains in the well infill (layer 2, sample $1 ; \mathrm{n}=92$ ). Secale cereale was dominant, but Hordeum vulgare, Triticum aestivum, and Panicum miliaceum were also documented. Half of the grains could only be determined as Cerealia. Overall, $92.4 \%$ of cereal evidence was conserved by carbonisation; only $7.6 \%$ was preserved by waterlogging.

Cereals formed $9.4 \%$ of the macroremains at the bottom of the well (layer three, sample two; n=55). Panicum miliaceum in the form of glumes was dominant; Hordeum vulgare and Secale cereale were documented as additional crops. Approximately $17 \%$ of the documented cereals were determined only as Cerealia. Only $25.5 \%$ of cereal evidence was conserved by carbonisation, $74.5 \%$ (especially millet glumes) were preserved by waterlogging.

\subsubsection{Other useful species}

A characteristic group of collected fruits were represented among other useful species; grown fruit and nuts were less common. Potential spices were frequently documented, and other useful species formed $5.92 \%$ of the documented macroremains in the well infill (layer two, sample $1 ; n=153$ ). Strawberries (Fragaria vesca, Fragaria sp.) were dominant, and raspberries (Rubus ideaus) and blackberries (Rubus fruticosus) were often documented. Blackthorn (Prunus spinosa, Prunus $\mathrm{sp}$.) and hazel (Corylus avellana) were rarely found. Figs (Ficus carica) were frequently documented as an imported fruit, and grown fruit were represented by apples and pears (Malus/Pyrus) and grapevines (Vitis vinifera). Potential spices could be hops (Humulus lupulus), which was the dominant useful plant in sample 1. Hemp (Cannabis sativa) was rare, and $97.4 \%$ of macroremains were preserved and waterlogged in sample 1.

Other useful species formed $18.1 \%$ of the documented macroremains at the bottom of the well (layer 3, sample 2, $\mathrm{n}=106$ ). Hops (Humulus lupulus) was a dominant potentially useful plant. Figs (Ficus carica) were more frequent than in sample one. Strawberries (Fragaria vesca), raspberries (Rubus ideaus), blackberries (Rubus fruticosus), blackthorn (Prunus sp.), hazel (Corylus avellana), and hawthorn (Crateagus sp.) were rarely found. Grown fruit was represented by apples and pears (Malus/Pyrus) and grapevines (Vitis vinifera), as well as in sample one. In sample $2,92.5 \%$ of macroremains were preserved and waterlogged.

\subsection{Charcoal and wood remains}

Two charcoal and wood samples were analysed (Figure 7B), and 9 taxons and 414 determinations were recorded (for list of determinations, see Table 1). The charcoal mass of infill well 1, layer 2 (sample 1) was $12,067 \mathrm{~g} / \mathrm{l}$, and the bottom well 1, layer 3 (sample 2) was $12,541 \mathrm{~g} / 1$. The bottom layer contained a large amount of wood fragments, and pine (Pinus sylvestris) had a dominant presence. A large number of spruce (Picea abies) wood was also recorded. Other wood fragments were identified as fir (Abies alba), oak (Quercus sp.), willow (Salix sp.), and occasionally beech (Fagus sylvatica). Many small pieces of rods and firewood charcoal pieces were contained in the bottom layer. Oak and pine charcoals were the most abundant; beech, fir, and willow charcoals were widespread; and birch (Betula sp.), hazel (Corylus avellana), and spruce were rare finds. The infill layer was characterised by a large amount of charcoal and only the small occurrence of wood fragments. This layer was distinguished by a large amount of pine charcoal pieces and frequent discoveries of beech, spruce, and fir charcoal. Only a small amount of pine, spruce, oak, beech, hazel, and willow wood fragments were found in this layer.

\subsection{Pollen analysis}

The pollen spectrum of sample 2 (well 1, layer three) consisted of 73 pollen types/families/genera, and 481 determinations were recorded (see Table 3). The spectrum was $89 \%$ nonarboreal pollen (NAP). Anthropogenic indicators (AI) dominated the spectrum.

A total of $32.2 \%$ consisted of cereals (Triticum type, Secale cereale, Hordeum type, Avena type, Cerealia type). Pollen grains of cornflower (Centaurea cyanus) comprised over $6 \%$, and poppy (Papaver rhoeas type) accounted for $1.3 \%$. Species of disturbed areas, such as knot-grass (Polygonum aviculare type), ruderals of the families Chenopodiaceae and Brassicaceae, weeds such as Anthemis arvensis type, and Rumex acetosa type, were the major pollen types in the sample. Species of Humulus lupulus type (both Humulus lupulus and Cannabis sativa) represented $1.9 \%$.

Overall, $9.2 \%$ of identified pollen grains belonged to Poaceae, and $6.24 \%$ were Ranunculus acris type (together with Ranunculus acris group). Plantago lanceolata type just exceeded the threshold of $1 \%$. Among arboreal pollen 
Table 3. Bakaláře Square, Písek, well 1, layer 3 (sample 2). List of pollen determinations.

\begin{tabular}{|c|c|c|c|}
\hline Pollen/NPP type & Count & Pollen/NPP type & Count \\
\hline Parasite egg & & Filipendula-type cf. & 1 \\
\hline Ascaris sp. & 22 & Geum-type & 1 \\
\hline cf Ascaris sp. & 1 & Hordeum-type & 2 \\
\hline Trichuris sp. & 30 & Humulus lupulus & 1 \\
\hline Arboreal pollen & & Humulus/Cannabis sativa & 4 \\
\hline Abies-type & 2 & Hypericum perforatum-type & 2 \\
\hline Acer campestre-type & 1 & Chenopodiaceae & 15 \\
\hline Alnus glutinosa-type & 2 & Lamiaceae & 1 \\
\hline Betula pubescens-type & 7 & Lathyrus/Viccia & 1 \\
\hline Calluna vulgaris & 1 & Lysimachia vulgaris-type & 1 \\
\hline Corylus avellana-type & 2 & Medicago lupulina & 1 \\
\hline Fagus sylvatica-type & 3 & Mentha-type & 2 \\
\hline Juniperus communis-type & 1 & Mercurialis sp. & 1 \\
\hline Picea-type & 5 & Microrrhinum minus & 1 \\
\hline Pinus sylvestris-type & 18 & Papaver rhoeas-type & 6 \\
\hline Quercus robur-type & 3 & Papaver sp. & 1 \\
\hline Salix-type & 2 & Peucedanum palustre-type & 3 \\
\hline Sambucus nigra-type & 3 & Plantago lanceolata & 5 \\
\hline Tilia cordata & 2 & Plantago media & 1 \\
\hline Nonarboreal pollen & & Poaceae & 44 \\
\hline Alchemilla pentaphyllea-type & 1 & Polygonum aviculare-type & 18 \\
\hline Anagallis arvensis-type & 3 & Polygonum cf. & 1 \\
\hline Anagallis arvensis-type cf. & 1 & Ranunculus acris-group & 18 \\
\hline Anthemis arvensis-type & 13 & Ranunculus acris-type & 12 \\
\hline Anthriscus sp. & 1 & Ranunculus acris-type cf. & 1 \\
\hline Apiaceae & 1 & Rumex acetosa-type & 5 \\
\hline Artemisia vulgaris-type & 3 & Rumex acetosella & 2 \\
\hline Asteraceae - Asteroideae & 2 & Rumex cf. & 1 \\
\hline Asteraceae - Cichorioideae & 4 & Sanguisorba officinalis-type & 1 \\
\hline Astragalus-type & 2 & Scleranthus annuus & 1 \\
\hline Avena-type & 2 & Secale cereale & 56 \\
\hline Brassicaceae & 13 & Senecio vulgaris-type & 1 \\
\hline Cannabis sativa & 4 & Silene dioica-type & 1 \\
\hline Carex-type & 2 & Sonchus oleraceus-type & 1 \\
\hline Carex $\mathrm{cf}$. & 1 & Taraxacum officinale-type & 2 \\
\hline Caryophyllaceae & 1 & Torilis japonica $\mathrm{cf}$. & 1 \\
\hline Centaurea cyanus & 29 & Triticum-type & 61 \\
\hline Cerastium fontanum-type & 3 & Urtica dioica-type & 1 \\
\hline Cerealia & 34 & Lemna-type cf. & 1 \\
\hline Convolvulus arvensis-type & 1 & Equisetum sp. & 2 \\
\hline Cyperaceae & 1 & Lycopodium sp. & 1 \\
\hline Daucus carota-type/Pimpinela major & 1 & Anthoceros sp. & 1 \\
\hline Dianthus superbus-type & 1 & Broken and corroded & 19 \\
\hline Filipendula-type & 2 & & \\
\hline
\end{tabular}

(AP), this threshold was only exceeded by pollen grains of pioneer species of pine (Pinus sylvestris type) and birch (Betula pubescens type), followed by spruce (Picea sp.). The total AP represented $11 \%$. Among other tree species, oak (Quercus robur group), beech (Fagus sylvatica type), lime (Tilia cordata type), willow (Salix sp.), hazel (Corylus avellana type), alder (Alnus glutinosa type), fir (Abies sp.), maple (Acer campestre type), and elder (Sambucus nigra type) were identified (Figure 7B).

Along with previously-mentioned grazing-indicator Plantago lanceolata type, pollen grains of juniper (Juniperus communis type), sheep sorrel (Rumex acetosella), and heather (Calluna vulgaris) scarcely appeared. Species of wet meadows as represented by, for example, Peucedanum 
Table 4. Bakaláŕe Square, Písek, wells 1 and 2, faunal spectra. NISP (Number of Identified Specimens), N (Number of Undetermined Specimens), MNI (Minimum Number of Individuals). The percentages are expressed as proportions of total remains.

\begin{tabular}{|c|c|c|c|}
\hline \multicolumn{4}{|c|}{ WELL 1} \\
\hline Faunal spectra & NISP; N & $\%$ NISP; $\% \mathrm{~N}$ & MNI \\
\hline Cattle (Bos taurus) & 8 & 5.76 & 3 \\
\hline Horse (Equus caballus) & 1 & 0.72 & 1 \\
\hline Domestic pig (Sus domesticus) & 2 & 1.44 & 1 \\
\hline Sheep (Ovis aries) & 4 & 2.88 & 2 \\
\hline Sheep/goat (Ovis/Capra) & 30 & 21.58 & 3 \\
\hline Dog (Canis familiaris) & 1 & 0.72 & 1 \\
\hline Domestic cat (Felis catus) & 9 & 6.47 & 1 \\
\hline Mouse (Mus sp.) & 7 & 5.04 & 2 \\
\hline Large and middle-sized mammals & 20 & 14.39 & - \\
\hline Very small rodent & 49 & 35.3 & - \\
\hline Undetermined mammal & 7 & 5.04 & - \\
\hline Undetermined fish & 1 & 0.72 & - \\
\hline Total & 139 & 100 & 14 \\
\hline \multicolumn{4}{|c|}{ WELL 2} \\
\hline $\begin{array}{r}\text { Faunal spectra } \\
\end{array}$ & NISP; N & $\%$ NISP; $\% \mathrm{~N}$ & MNI \\
\hline Cattle (Bos taurus) & 3 & 15.79 & 1 \\
\hline Domestic pig (Sus domesticus) & 6 & 31.58 & 2 \\
\hline Sheep/goat (Ovis/Capra) & 1 & 5.26 & 1 \\
\hline Hare (Lepus europaeus) & 1 & 5.26 & 1 \\
\hline Large and middle-sized mammals & 8 & 42.11 & - \\
\hline Total & 19 & 100 & 5 \\
\hline
\end{tabular}

palustre type, Filipendula type, Cyperaceae/Carex, Pimpinella major type, Lysimachia vulgaris type, and Sanguisorba officinalis, belonged to the "rare occurring species/types" in the sample. The rare presence of other weeds was confirmed, e.g. Microrrhinum minus, Scleranthus annuus type, Anagallis arvensis type, Artemisia vulgaris type, Convolvulus arvensis type, and Sonchus oleraceus type.

\subsection{Parasitology}

Presence of parasite eggs was confirmed in the sample based on the concentration technique and flotation method. Three genus of helminths were identified: Capillaria sp., Ascaris sp., and Trichuris sp. Moreover, the presence of intestinal parasite eggs was recorded along with the pollen, which enabled us to estimate their abundance. Besides the 481 total pollen counts, eggs of the genus Ascaris sp. and Trichuris/ Capillaria sp. reached 22 and 30 counts, respectively $(9.8 \%$ parasite eggs on TPS and NPP).

\subsection{Archaeozoology}

Well-preserved osteological material was found in both wells, and 158 faunal remains were recorded in total. Remains of domestic fauna were prevalent, and the element representation was varied (among described specimens, e.g. fragments of skulls and jaws, long bones, autopodials, ribs, shoulder blades, and pelvis).

Well 1: All the determined finds (little in layer 1 and 3, many in layer 2) reflected the presence of domestic animals
(88.7\% of NISP); cattle (Bos taurus), horse (Equus caballus), sheep (Ovis aries) and alternatively goats (Capra hircus), pigs (Sus domesticus), dog (Canis familiaris), and cat (Felis catus). Several bones of postcranial skeletons of small rodents, e.g. mice (Mus sp.) were found only at the bottom of the well (layer 3, sample 2). From the total number of 139 bones and teeth, $44.6 \%(\mathrm{NISP}=62)$ of specimens found were taxonomically identified. The rest of the specimens (55.4\%; NISP=77) were identified as large or middle-sized mammals, very small rodents and undetermined mammals. Among the unidentified part of the assemblage (layer 2, sample 1) a fragment of damaged scale of fish was also present. The archaeozoological data are assumed in Table 4.

Most of the bones were dark brown in colour, which reflects the anaerobic conditions in that part of the well where the animal bones were deposited in the $14^{\text {th }}$ century. The astragalus bone of an adult horse had weathering cracks and was found in layer 1 of well 1. It was the only part of the animal found in both well 1 and within the rest of the salvage excavation of medieval contexts at the Bakaláre square, Písek.

In the narrowing part of well 1 (lower part of layer 2) an incomplete adult bull horn was found. The porous fragment of the frontal bone belonged to a juvenile (calf), as well as a fragment of left jaw bone found in the upper part of layer 2 . The nearly complete hornless skull of a sheep older than 6 years was also uncovered. The second sheep in the archaeozoological collection was determined by a partly 
damaged jaw bone of the slaughtered animal in the lower part of layer 2. If the jaw bone was connected with the skull, mentioned above, is unclear. Some parts of caprines ("goatssheep") were not distinguished, but specimens included two tibia bones with different degrees of epiphyseal fusion. The specimen drawings confirm that these must be from two individuals. The individual age of one of them was less than 3 years, and the second animal was older. The other sheep/ goat bones (parts of fore and hindlimbs), deposited in the upper part of layer 2, pointed to osteological material from a third, physically immature, individual (less than 3 years old). Teeth, believed to be from a pig, belonged to an animal killed between 12 and 18 months old. In some cases, the butchery marks on the bones were visible. The cut marks were noticed on meat-bearing bones, e.g. on ribs of caprines, ischium of cattle, and shoulder blades of a large-sized mammal. A slightly damaged thoracic vertebra of an adult dog and part of an adult cat skeleton (including pelvis, some long limb bones, and ribs) were recorded by osteological analysis. Almost all of the carnivore bones were darkly coloured, with the exception of the light-coloured radius of the cat, which probably belonged to the aforementioned skeleton.

Well 2: The archaeozoological collection was obtained from the test probes in the upper layer of well 2. The identification of the bones in this sample confirmed the presence of one cattle, two pigs, one sheep/goat, and one hare (Lepus europaeus), which was the only wild animal in the whole assemblage (Table 4). From the total number of 19 bones, teeth, and their fragments found, $57.9 \%(\mathrm{NISP}=11)$ of the specimens were taxonomically identified. The exploitation of pigs for consumption was supported by the observation that a third of the bones had butchering marks on their surface. The pigs were slaughtered at an immature age, before reaching 2 years old. The domestic animals were represented both by meat-bearing bones and fragments of the skulls or teeth. The first phalanx of cattle found in well $2 \mathrm{had}$ been modified to make a tool.

\section{Discussion}

Archaeological and bioarchaeological evidence from the investigation of the well infill can partly answer the questions of infill origin and well utilization.

\subsection{Dating of the backfill based on ceramic chronology and dendrochronology}

Dating and placing of pottery fragments from the surface of well 1 (layer 1) and well 2 (layer 1) is identical. However, we could only define and compare the chronologically-latest backfill on the surfaces. We do not know whether both wells performed their primary function at the same time.

Some of the pottery fragments could be identified as imported vessels associated with long-distance trade contacts or the presence of foreign ethnic groups (colonists and specialised craftsmen, coming from the area of presentday Germany and Austria). Analogous thin-walled vessels made of light clay (Figure 6: 9), are known from a medieval cesspit in Most (Klápště 2002, 21-22, Figures 123: 4, 144 : 3 ) dated to the $14^{\text {th }}$ century.

Graphite pottery from Bohemia is typical for the $13^{\text {th }}-15^{\text {th }}$ centuries, although it is not chronologically sensitive. Ceramics made with a mixture of graphite could be used to process and transport tar; as the crusts on the walls of these vessels confirmed.

No entire vessels were found; this shows that only fragments were thrown into the well. In ceramic collections from wells, datable finds in a broader chronological section are quite common phenomena in an urban environment. It reflects the complex of processes that shaped the archaeological terrain in medieval towns (generally Nováček 2003, 131-146; Čapek 2010).

The pottery here can be dated on the basis of analogies from urban and rural settlements from the south Bohemian area and elsewhere (Reichertová 1965; Richter, Krajíc 2001; Procházka 1994; Nekuda, Reichertová 1968; Radoměrský, Richter 1976; Vařeka 1998, Orna ed. 2011), to the middle of the $14^{\text {th }}$ century. A few pottery fragments of vessels found at the bottom of the well were also dated to the middle of the $14^{\text {th }}$ century. Based on dendrochronological dating, the well was backfilled after the year 1318, which is consistent with the dating of the ceramics. Due to the fact that the town was founded around 1243, the well could have been used for its primary function (water reservoir) for at least 100 years.

\subsection{Reconstruction of the economy and environment at the time of the wells infilling - character of the infill}

The advent of location and institutional towns brought a footprint on the behaviour of the population. The urban area was divided into fixed property parcels - and the rights and obligations of the population related to their possession of these plots. Various aspects of human life became adapted to the new framework. In archaeology, the changes are reflected in waste disposal and storage (Klápště 2005, 388; Hoffmann 1992, 331).

The accumulation of household waste, which often ended in the street, was a problem. There were also complications associated with agricultural activities (breeding of domestic animals or cultivation of plants) in the town. Some handcrafts were banished beyond the town walls for their production of impurities (pollutants) and odours, while others stayed within the towns. In particular, butcher waste caused substantial hygienic problems (Hoffmann 1992, 332). Various methods of urban waste deposition were evident. Sometimes a series of pits were used, sometimes items were consistently thrown into or taken away by the river (Klápště 2005, 392).

The wider urban area included both the fortified core of the town and the suburbs, where agricultural land cultivated directly from the town, and gardens and vineyards, were included.

The influence of several towns reached into nearby villages (Hoffmann, 1992, 82). Urban development was associated with relations and contacts between the urban 
community and its regional and supra-regional contexts. The agricultural hinterland of towns brought a benefit both for the towns and villages, and at the same time deepened the relation between them (Klápště 2005). The different raw materials and products were transported to the towns as a result of these contacts, and their remains and leftovers were subsequently deposited as waste.

The application of multiple bioarchaeological methods helped to reconstruct and interprete the infill of the well, even though the analyses were applied to only two samples. We partly managed to reconstruct the environmental conditions of the town and its surroundings, as well as the economic background of the town (waste management, food strategy, animal husbandry, and exploitation of natural resources).

We suggest that in the case of a feature with a clear, simple stratigraphy, and when salvage excavations are limited by proper financial support, it is better to include several types of analyses to a low number of samples, rather than doing one type of analysis on many samples based on systematic sub-sampling. Our results imply that the socio-economic reconstruction of the medieval town can be fully reliable in such cases.

The investigated feature (well 1) could be interpreted as a secondary backfilled well. The character of the bottom, layer 3, cannot be considered as pure faecal waste (after Smith 2013). However, faecal inclusion should be taken into consideration due to the presence of the eggs of intestinal parasites, which was confirmed by parasitological investigation. This result was supported by relatively high amounts of Ascaris, Trichuris, and Capillaria egg remains recorded on the pollen slides. The presence of fruit macroremains (ca 8\%) could support the assumption of its faecal origin. But, their abundance was low in comparison to the high proportions of segetals/ruderals (more than 50\%) and species of wetlands and meadows ( $c f$. Figueiral, Séjalon 2014; Florenzano et al. 2012).

The recovered macroremains and the pollen spectrum from layers 2 and 3 probably reflect waste plant material of various origins. The occurrence of plant species typical for meadows could indicate the presence of hay residues and the issue of the storage and transportation of such grasses into the town (Kosňovská et al. 2011), and secondarily stored inside well 1 after the loss of its primary function. Such species compositon of the well infill most likely reflects the plant material (macroremains) that was brought into the town from different surrounding habitats (floodplains and fields are the most obvious; $c f$. Figueiral, Séjalon 2010).

Based on the stratigraphy and results of the bioarchaeological analyses, we can interpret the formation of the three layers of well 1. Layer 3 was dark and muddy with sand and was deposited at the bottom of the well. It is possible to interpret this layer as primarily sediment in the period of the ending of its function as a well, when the water was partly contaminated by waste. Its macroremain concentration is much lower than in layer 2, and the absence of diatom frustules in the samples examined suggests either a terrestrial origin for the sediment or inappropriate living conditions for diatoms in an existing aquatic environment. The latter could have been caused by a limited access of light into the water column e.g. the use of a hatch. In the third eventuality, there could have been dissolution of silica-built diatom frustules after its deposition and this should also be taken into account, although the high sediment-carbonate content predicative of such a process was not recorded.

Parasite eggs observed in the sample from layer 3 could have been produced by parasites from animals, as well as from humans. Ascaris sp. and Trichuris sp. eggs are the most common in paleoparasitological findings (Goncalves et al. 2003). The genus Ascaris has two common species: A. lumbricoides in human and $A$. suum in pigs. Due to the morphological similarity of both species' eggs, their origin could not be determined. The same problem occurs with Trichuris sp., which parasitise humans (T. trichiura), pigs, and also dogs. Eggs of Capillaria sp. (roundworms) were very abundant in the sample, which are parasites of domestic animals and rodents. Human infection by this parasite is very rare but possible. Faecal origin can be attributed to the waterlogged macroremains of strawberries (Fragaria vesca, Fragaria sp.), raspberries (Rubus ideaus), blackberries (Rubus fruticosus), blackthorn (Prunus spinosa) and figs (Ficus carica); as well as these fruits, kitchen waste is also a possibility ( $c f$. Smith 2013; Bosi et al. 2009). Different processes revealed charred grains, which were probably charred before their grinding/milling inside the medieval town (kitchen waste/burning waste; see, for example, Petráň 1985, 389-394). Waterlogged glumes of Panicum miliaceum are evidence of grain cleaning before processing in the kitchen. Other groups of macroremains (Table 1) and the pollen spectra (Table 3) document the different origins of infill: fields, rubble, forests, wetlands, and meadows, which all reflect the town's economy. The presence of waste, originally coming from plants probably growing in fields, rubble, forest, wetlands and meadows, could be a reflection of this (cf. Figueiral, Séjalon 2010).

Dirty water from the well was not ideal to drink, so the well could have lost its primary function and eventually became a cesspit.

Layer 2 was made up of black muddy sediment and was deposited from the depth of 4 to 7.6 metres after the year 1318 A.D. The concentrations of macroremains per litre in layer 2 were much higher compared with layer 3 . As with layer 3, layer 2 also contained waste of various origins, such as fields, rubble, forests, and wetlands; however, this deposition of waste was more concentrated. The proportions of cereal and useful plants were much lower compared with those in layer 3 (Tables 1 and 2). The character of the archaeozoological collection could be defined as waste, being the remains of consumed animals and dead house animals (Table 4).

Layer 2 can be interpreted as intentional waste storage in the period after the end of the well's primary function, and it is possible to identify this layer as secondary waste sediment.

Layer 1 consisted of fragments of daub, often carrying the construction imprints, and large iron slags. Based on X-Ray 
Fluorescent Analysis findings it was impossible to determine the origin of the daub. It is possible to interpret layer one as intentional secondary waste storage, perhaps some destroyed production equipment related to metal processing.

\subsection{Animal remains}

In the archaeozoological collection of two medieval wells, according to the representation of bone elements, taxonomic identification, and butchery marks, we can assume that the remains of consumed herd animals (e.g. cattle, sheep, and pig), house animals (dog or cat), animals used in working activities (horse), and pests (small rodents), were deposited in non-functional wells. The animal remains were partially protected from weathering and scavengers. The animal bones and teeth were discarded in anaerobic conditions, and they remained well preserved. The analysis confirmed that the inhabitants were involved in animal husbandry; hunting was suggested by one bone from a hare. Despite the low frequency of bone remains found in the wells, the main source of meat and other animal products in well 1, were sheep or goats, less so cattle. Conversely, the bones and teeth of pigs prevailed in well 2. The slaughter of livestock, both younger and adult individuals, for meat was evidenced by the butchery marks on some bone surfaces. The elder individuals were presumably used for secondary products, e.g. milk or wool (in the case of sheep). The use of animal bones (more specifically the first phalanx from cattle) as a raw material for the making of tools was likewise confirmed. The distributions of body parts did not reveal any differences between the wells. The collection of animal remains can be considered as waste, as bones from the meaty parts of animals did not outnumber those parts usually separated out during butchering, such as hooves and heads. The bone refuse had obviously been mixed in both wells, no significant differences being found in the rate of fragmentation between the two wells.

\subsection{Pollen}

The pollen spectra can be considered as being typically medieval for the contexts of the Czech Republic. The combination of high amounts of pollen grains of Secale cereale and Centaurea cyanus is considered to be an indicator of High Medieval sediments (e.g. Jankovská 1997; Kočár et al. 2010; Kozáková et al. 2014), and the occurrence of Centaurea cyanus was proved to be an indicator of High Medieval sediments based on the dataset originating from medieval Prague, Czech Republic (Kozáková et al. 2009).

The low relative abundance of trees and the high relative abundance of ruderals and useful plants in the bioarchaeological record corresponded with the character of the well infill. Its deposition was human made and caused by the specific economical requirements of the material. Its primary function was most probably as feed or bedding. Low amounts of arboreal pollen (AP) could correspond to a high level of deforestation in the area, but the ratio is usually underestimated in the case of well infill. The comparison with the AP/NAP ratio from a sample of approximately the same era (around 1350 A.D.) originating from a nearby core taken from a relatively large water area, the fishpond in Řežabinec, shows a discrepancy (Rybníčková, Rybníček 1985). The proportion of AP there was much higher, at around $45 \%$. This implies that the infill of the well reflects taphonomic processes affected by the direct accumulation of waste. The composition of trees and shrubs was similar to the species composition originating from the fishpond in Řžabinec. The dominance of pine and birch was similar; however, a lower proportion of spruce was detected in the case of the well bottom's pollen spectrum, as the spruce pollen percentage has continuously increased in the surroundings since the High Medieval period. An even higher AP ratio $(72.6 \%)$ was recorded in small spring mire in Kožlí situated 10 km northwest of Písek (Pokorný, Kuneš 2009). Despite the relatively vast chronological interval of the layer corresponding to the time period of our interest, the given vegetation reconstruction is characterised by high tree ratios, especially of pine and spruce. In the core taken from the Smutná River floodplain near Sepekov, situated $25 \mathrm{~km}$ northeast of Písek, arboreal pollen composed one-half of the recorded spectrum (Houfková et al. 2011).

This information supports the idea of the well pollen spectrum having been affected primarily by taphonomic processes rather than direct waste accumulation.

The bottom, layer 3, would reflect the presence of waste of local origin with a low admixture of bedding waste. The composition of the pollen spectrum consisted mainly of cereal species and their weeds. As the analysis of fossil material recovered from non-specific urban archaeological sites has proved repeatedly, the real, local mosaic of habitats and vegetation is reflected in the recovered spectra (SwietaMusznicka et al. 2013); species of local disturbed habitats could partially contribute to the species composition of the analysed sample (e.g. species of the family Chenopodiaceae, Brassicaceae, or of pollen types such as Rumex acetosatype, Polygonum aviculare-type). The relatively minor portion of the pollen spectrum represented by species of the family Poaceae, and species included in the pollen types Peucedanum palustre type, Filipendula type, Cyperaceae/ Carex, Pimpinella major type, Lysimachia vulgaris type, and Sanguisorba officinalis, could imply that its origin was periodically-inundated alluvial wet meadows. The scarce occurrence of subfossil seeds of Carex disticha or Eleocharis sp. would support such a scenario.

However, the spectrum of plant species from layer two, as recovered by the macroremains analysis, would originate from alluvial meadows characterised as tall-sedge beds. That could be interpreted as the presence of hay residues and could suggest the presence of bedding or feed waste.

The species composition of arboreal pollen was comparable to the species spectrum recorded by the antracological analysis (Figure 7B).

\subsection{Charcoal and wood remains}

The large number of fragments of wood and rods suggest the presence of waste material in the bottom sediment layer. The wood fragments probably come from the processing of 
structural timber. This bottom layer also contained firewood charcoal. The source of fuel wood is usually interpreted as being local, whereas construction wood is given as an imported material. Medieval wood imports were widely carried out by floating (e.g. Beneš et al. 2006).

However, the species composition of the pollen samples reflected a similar species composition to that of the surrounding forests (vegetation). The possible local origin of structural timber would support the high proportion of spruce and pine pollen. The infill layer contains significantly more pieces of charcoal. The species composition is more or less unchanged from the bottom layer, but the quantity of typical firewood species increases over time. It shows a higher presence of beech, birch, and hazel charcoal pieces. The firewood was probably formed by wood-processing residues, local wood resources, and wood imports from the surrounding area. The anthracological data reconstruct the forest vegetation as being acid oak with beech forests in the vicinity. This reconstruction almost corresponds to a map of potential vegetation for this area that reconstructs bird cherry-pedunculate oak and alder woodland partly in a mosaic with alder carrs, reed swamps, and tallsedge communities (Quercus robur-Padus avium, Alnus glutinosa-Padus avium with Carex brizoides, locally with Carici elongatae-Alnetum and Phragmito-Magnocaricetea) (Neuhäselová 2001).

\subsection{Plant macroremains}

The results of the pollen analysis from the samples originating from the well infill sediment compared to the samples originating from the natural pollen profiles reflect different environments and a complex of taphonomic processes.

Different processes are also reflected in the infill of the well by the charred or waterlogged macroremains. A comparison of the results of the analysis of pollen and plant macroremains provides a reflection of these different processes (Figure 7A). Macroremains reflect the waste from crop processes (ruderals/segetals, weeds, and cereal weeds), which could be used secondarily in the economy of the medieval town (litter, animal feed, building material, etc.), and kitchen waste (glumes of millet, charred grain). Macroremains typical for meadows and floodplains could reflect the litter and animal feed. In connection with the findings of numerous hops seeds, it is possible that the inhabitants produced beer. This could also be suggested by the infrequent sprouted barley caryopses related to malting (Kočár et al. in print). Written sources for malting in Medieval Písek, however, do not exist.

The spectrum of cereals captured in the well infill (Secale cereale, Hordeum vulgare, Triticum aestivum, Panicum miliaceum) is comparable with all standard quantified results in the Czech Republic (Kočár et al. 2010, Kočár et al. in print) and central Europe, typical for the Middle Ages. However, for typical faecal pits, the concentration of carbonised grain is not common (for the region of South Bohemia see, for example, Pokorný et al. 2002; Opravil in Krajíc et al. 1998, 200-204; Čapek et al. in print), because most grain was milled outside town centres; home grinding was more frequent in the villages (Petrán̆ 1985, 389-394). We thus believe that the well infill reflects more the origins of the waste.

Other useful species - collected fruits, grown fruits, nuts, and potential useful spices - could reflect a local food strategy and other areas of resource collection, but they could also be traded goods. Long-distance trade and regional markets prospered in the Czech Lands in the Middle Ages (Klápště 2005, 361). Medieval Písek was situated on one of the trade routes from the south to Prague (Fröhlich 2013). Strawberries (Fragaria vesca, Fragaria sp.), raspberries (Rubus ideaus), blackberries (Rubus fruticosus), blackthorn (Prunus spinosa, Prunus sp.), hazel (Corylus avellana), and hawthorn (Crataegus sp.) may all reflect an area of cleared forests and their edges. Grown fruit was represented by apples/pears (Malus/Pyrus) but was rare; we would have expected fruit trees to have been grown at the back of the town (Hoffmann 1992, 144). Fruit was used fresh, dried or in the form of jam or alcoholic beverages (Hoffmann 1992, 342). The issue of growing hemp (Cannabis sativa) and hops (Humulus lupulus) is complicated; it is impossible to determine whether the seeds were cultivated or secondary wild plants, but their use in the medieval economy is probable (e.g. hemp oil: Hoffmann 1992, 343). Seeds from grapevines (Vitis vinifera) document either trade or farming. Figs (Ficus carica) were almost certainly imported, which suggests regular long-distance trade between central and southern Europe (Hoffmann 1992, 342). The growing of figs in the climatically-favourable conditions of towns could be expected, but for Písek it is not supported by any written documentation. Figs had an important role in the human diet as a source of sugar (Figueiral, Séjalon 2010). Alluvial wet meadows, which were noticeably reflected in the plant macroremains and floodplains, could be expected in the area immediately behind the town by the Otava River. We would expect irregular mowing and the gathering of such resources, especially for animal husbandry (feed, litter), in the immediate surroundings. Dry meadows, which are also highlighted by the plant macroremains, could have been similarly used as were the alluvial wet meadows.

Buds and needles could, like the macroremains of plants typical for meadows, indicate animal husbandry and also building material (Quercus sp., cf. Populus tremula, Pinus sp., and Abies alba). Needles of Juniperus communis may indicate grazing, and the buds of trees suggest that the dried twigs and shoots were used as feed in winter.

\section{Conclusion}

By processing the two medieval wells excavated in 2008 in Písek, Bakaláře Square (South Bohemia, Czech Republic), we were able to apply several different bioarchaeological methods combined with traditional analyses of artefacts.

Among the methods of bioarchaeology employed were the analysis of plant macroremains, analysis of wood and 
charcoal, pollen analysis, dendrochronology, archaeozoology, paleoparasitology, and diatom analysis. Additionally, many types of artefacts were analysed (ceramics and wood, fragments of glass, slag, and daub).

Various aspects of human life and behaviour in medieval Písek were reflected in the waste disposal and storage found inside wells 1 and 2. It was possible to detect imported material of different origins and to reconstruct the environment of the town's background (meadows, fields, gardens, forests, etc.), as well as animal breeding (e.g. cattle, sheep, pig, horse, dog and cat), growing of crops (e.g. cereals and fruits), and waste management practices.

The infill of the wells was dated to the $14^{\text {th }}$ century based on dendrochronology and artefact typology. Well 1 and well 2 were definitely backfilled probably in the $14^{\text {th }}$ century. Well 2 was not excavated entirely; however, the sediments were conserved for future research with better scientific possibilities.

The position of the two wells, whose original purpose was apparently to supply water, in the mutual vicinity represents for current scholars an unclear situation. Perhaps it reflects a former division of medieval alotments, or perhaps they were dug succesively. The dating of the infilling of the wells, which, unfortunately, can only be estimated very imprecisely as one being close to the other, does not allow for a clearer conclusion. After some period of use both wells were filled in; the reason for this is also unclear. The character of the infill is known only in the case of the first well, which was excavated in its entirety. The first part was mostly filled by a layer, the origin of which could be loosely imagined as the result of a combination of accumulating animal bedding/ dung and kitchen waste - with occasional "sweepings" of carcasses. This "unattractive" mass was then covered by a layer formed probably by the broken up remains of some large manufacturing production device that could possibly be ascribed to a furnace for smelting. The active use of such a device within the city-walls could be considered a surprise. On the other hand, the site is located in the close neighbourhood of the church, which could be imagined as being still partly in construction during the first half of the $14^{\text {th }}$ century.

The analyses of the both upper and lower part of the sediments from well 1 have brought a suite of information: a wide range of artefacts and ecofacts that not only fill taxonomical and analogical lists. It has also brought much information about the spatial relatioships of the site within the microworld and macroworld of a medieval town. In this case, the archaeogeographical relationships can be divided into three major categories. The first should include the evidence formed by the fragments of slag and daub that probably reflects the relations of people and local industry/ trade within the town-wall. Into this category should probably also belong the finds of some of the animal bones that were kept with households (goats, pigs, cats, dogs, etc.). The second category refers to the origin of the plants species - those used for the feeding and bedding of animals, for which those of wetlands and meadows are characteristic. The same is valid for the sources of firewood, and the rest of the more mobile animal species (horse, cattle, etc.). This group reflects the interactions between the town and its economical hinterland outside the town-wall. Only in this case could the classical von Thünen's zones analyses (von Thünen 1826) for regional and municipal economies be eventually taken into account. The last category is formed of the isolated examples of "imports" that probably came from beyond the region's borders. Among these, the examples of figs could be mentioned. Further, the example of the fragment of a thinwalled vessel made of light clay and graphite ceramics could have played an important role.

\section{Acknowledgments}

This project was supported by GAJU 116/2013/P, by the project "PAPAVER - Centre for human and plant studies in Europe and Northern Africa in the postglacial period", reg. No. CZ.1.07/2.3.00/20.0289, co-financed by the European Social Fund, and the state budget of the Czech Republic. This article was also supported by the project Postdoc USB (reg. no. CZ.1.07/2.3.00/30.0006) realized through the EU Education for Competitiveness Operational Programme, by the NEXLIZ CZ.1.07/2.3.00/30.0038 and GA CR P405/10/0556. Special thanks are due to Jindřich Kurz, Jiří Bumerl, Václav Holeček, Zdeněk Mužík, Zdeněk Vaněček, and the staff of Technical Services of Písek for technical support during the excavation of well 1 . We would like to thank archaeologists Eva Koppová and Jiří Fröhlich for their many years of research in the historical centre of the town and their expansion of what is known concerning water management in the area of medieval Písek. We are grateful to Jan Adámek for information about education in medieval Písek and Jaroslav Frána for performing the RFA analysis. We are also deeply grateful to Věra Klontza Jaklová, Sabine Karg and Anna Maria Mercuri for their constructive criticism of earlier versions of this paper.

\section{References}

ADÁMEK, J., FRÖHLICH, J.: 2002. Archeologický výzkum zaniklého dominikánského kláštera v Písku (III). Prácheňské museum v Písku. Zpráva o činnosti za rok 2001, 33-40.

ANDERBERG, A. L. 1994: Atlas of seeds and small fruits of NorthwestEuropean plant species with morphological descriptions. Stockholm: Swedish Museum of Natural History.

BATTARBEE, R. W. 1986: Diatom analysis. In: Berglund B. E. (Ed.) Handbook of Holocene Paleoecology and Paleohydrology. Wiley and Sons, Chichester, 527-570.

BENEŠ, J., KOLÁŘ, T., ČEJKOVÁ, A. 2006: Xylotomic and Dendrochronological Analyses in Archaeology: Changes in the Composition Type of Wood in Prague and in Southern Bohemia. Ve službách archeologie VII, 159-169.

BERGGREN, G. 1981: Atlas of seeds and small fruits of Northwest European plant species with morphological descriptions. Swedish Museum of Natural History, Stockholm.

BEUG, H. J. 2004: Leitfaden der Pollenbestimmung für Mitteleuropa und angrenzende Gebiete. München. 
BOSI, G., MERCURI, A. M., GUARNIERI, C., BANDINI MAZZANTI, M., 2009. Luxury food and ornamental plants at the $15^{\text {th }}$ century A.D. Renaissance court of the Este family (Ferrara, northern Italy). Vegetation History and Archaeobotany 18 (5), 389-402.

CALLEN, E. O, CAMERON, T. N. 1960: A prehistoric diet as revealed in coprolites, New Scientist 8, 35-39.

CAPPERS, R. T. J. et al. 2006-2009: Digital Seed Atlas of the Netherlands. Groningen: Groningen Institute of Archaeology and the Deutsches Archäologisches Institut.

ČAPEK, L. 2010: Depozični a postdepozični procesy na parcelách Českých Budějovic. (prípadová studie z doти čp. 16). Keramika - KvantifikaceStatistika-Chronologie. Plzeň.

ČAPEK, L., CHVOJKA, O., KODÝDKOVÁ, K., HOUFKOVÁ, P., MYŠKOVÁ, E., BENEŠ, J. in print: Středověká odpadní jímka domu čp. 7 v Krajinské ulici v Českých Budějovicích ve výsledcích rozboru artefaktů a environmentálních analýz. Archeologické výzkumy v jižních Čechách.

FAEGRI, K., IVERSEN, J. 1989: Textbook of Pollen Analysis. Chichester.

FIGUEIRAL, I., SÉJALON, P. 2014: Archaeological wells in southern France: Late Neolithic to Roman plant remains from Mas de Vignoles IX (Gard) and their implications for the study of settlement, economy and environment. Environmental Archaeology 19/1, 23-38.

FLORENZANO, A., MERCURI, A. M., PEDERZOLI, A., TORRI, P., BOSI, G., OLMI, L., RINALDI, R., BANDINI MAZZANTI, M. 2012 The Significance of Intestinal Parasite Remains in Pollen Samples from Medieval Pits in the Piazza Garibaldi of Parma, Emilia Romagna, Northern Italy. Geoarchaeology 27, 34-47.

FOTT, B. 1954: Pleurax, synthetická pryskyřice pro preparaci rozsivek. Preslia 26, 163-194.

FRÖHLICH, J., KOPPOVÁ, E. 1995: Výzkum středověké studny domů čp. 118 a 119 v Písku. Jihočeský sbornik historický 64, 3-19.

FRÖHLICH, J., KOPPOVÁ, E. 2000: Archeologický výzkum v Písku v roce 1999. Prácheňské muzeum v Písku. Zpráva o činnosti za rok 1999, $32-38$.

FRÖHLICH, J. 1997: Pisecko v zrcadle archeologie. Písek.

FRÖHLICH, J. 2002: Středověké odpadní jímky v Písku. Archeologické výzkumy v jižnich Čechách 15, 139-161.

FRÖHLICH, J. 2013: Pokus o rekonstrukci středověkých dálkových komunikací kolem královského města Písku. Výběr. Časopis pro historii a vlastivědu jižních Čech 50/4, 272-275.

GONCALVES, M. L. C., ARAÚJO, A., FERREIRA, L. F. 2003: Human intestinal parasites in the past: New findings and a review. Mem Inst Oswaldo Cruz 98, 103-118.

GRANT, A., 1982: The use of tooth wear as a guide to the age of domestic ungulates. In: Wilson, B., Grigson, C., Payne S. (Eds.), Ageing and sexing animal bones from archaeological sites. British Archaeological Reports. International Series 109, 91-108.

GRIMM, E. C. 2011: Tilia. Ver. 1.5.12. Springfield: Illinois State Museum.

HEISS, A. G. 2000: Anatomy of European and North American woods. An interactive identification key.

HEJNÝ, S., SLAVÍK, B. (Eds.) 1988-1992: Květena České republiky. Vol 1, 2, 3. Praha, Academia.

HELMER, D., 1995 : Biometria i arqueozoologia a partir d'alguns exemples del Proxim Orient. Cota Zero 11, 51-60.

HELMER, D., VIGNE, J. D. 2004: La gestion des cheptels de caprinés au Néolithique dans le midi de la France. In: Bodu, P., Konstantin, C. (Eds.): Approches fonctionnelles en Préhistoire (Actes XXVe Congrès Préhistorique de France Nanterre, 24-26 novembre 2000). Paris: Société Préhistorique Française Édition, 397-407.

HOUFKOVÁ, P., BERNARDOVÁ, A., NOVÁKOVÁ, K., NOVÁK, J 2011: Sepekov - vodárna Závist: záznam mladoholocenní vegetační dynamiky jedlové olšiny. Rekonstrukce vegetačních změn v nivě říčky Smutná u Sepekova. In: Chvojka, O. et al.: Osídlení doby bronzové v povodi ríčky Smutné v jižnich Čechác. Archeologické výzkumy v jižnich Čechách. Supplementum 8. České Budějovice, 328-333.

HOUFKOVÁ, P., JIŘÍK, J., KOVAČIKOVÁ, L., PTÁK, M., ŠÁLKOVÁ, T. 2013: Medieval and Modern Water Management. An example from the excavation in Písek - Bakaláře-square (Southern Bohemia). In: Fines Transire Jahrgang 22, 163-172.

JANKOVSKÁ, V. 1997: Výsledky pylových analýz z lokality: Praha 1 Malá Strana, Tržiště 259/III. In: Kubková, J., Klápště, J., Ježek, M.,
Meduna P. (Eds.): Život v archeologii stredověku. Archeologický ústav AVČR, Praha, 299-308.

JIŘÍK, J., PTÁK, M. 2010: Archeologický výzkum v dome čp. 94 na Havlíčkově náměstí v Písku. MS: Unpublished report of archaeological survey. Deposited: Prácheň Museum in Písek.

KLÁPŠTĚ, J. 2002: Archeologie středovékého domu v Mostě (čp. 226). Praha, Archeologický ústav AV ČR.

KNIBBE, B. 2004: Past4 - Personal Analysis System for Treering Research Version 4, Instruction Manual, SCIEM/Bernhard Knibbe, Wien.

KOČÁR, P., ČECH, P., KOZÁKOVÁ, R., KOČÁROVÁ, R. 2010: Environment and Economy of the Early Medieval Settlement in Žatec. Interdisciplinaria archaeologica. Natural Sciences in Archaeology $\mathrm{I} / 1-2 / 2010,45-60$.

KOČÁR, P., BENEŠ, J., PREUSZ, M., VANĚČEK, Z. in print: Ječmen a ječný slad ve středověku a raném novověku v českých zemích. Kvasný průmysl.

KOSŇOVSKÁ, J., HOUFKOVÁ, P., KOVÁŘ, D. 2011: Lannova tř́ída ve světle archeobotanických analýz. Archeologické výzkumy v jižních Čechách 24, 263-280.

KOZÁKOVÁ, R., POKORNÝ, P., HAVRDA, J., JANKOVSKÁ, V. 2009: The potential of pollen analyses from urban deposits: multivariate statistical analysis of a data set from the medieval city of Prague, Czech Republic. Vegetation History and Archaeobotany 18, 477-488.

KOZÁKOVÁ, R., POKORNÝ, P., MAŘÍK, J., ČULÍKOVÁ, V., BOHÁČOVÁ, I., POKORNÁ, A. 2014: Early to high medieval colonization and alluvial landscape transformation of the Labe valley (Czech Republic): evaluation of archaeological, pollen and macrofossil evidence. Vegetation History and Archaeobotany 23, 701-718.

KRAJÍC, R. et al. 1998: Di̊m pasíre Prokopa v Táboře (Archeologický výzkum odpadní jímky v domě čp. 220). Tábor.

KREUZ, A., SCHÄFER, E. 2002: A new archaeobotanical database program. Vegetation History and Archaeobotany 11, 177-179.

KUDRNÁČ́, J. 1971. Zlato v Pootaví. Písek.

NECHVÁTAL, B. 1967: K nálezové problematice středověkých studní a odpadních jímek. Český lid 54, 170-174.

NECHVÁTAL, B., SMETÁNKA, Z. 1965: K interpretaci odpadních jímek a studní v městském prostředí. Archeologické rozhledy 17, 262-263, 56.

NEKUDA, V., REICHERTOVÁ, K.1968: Středovéká keramika v Čechách a na Moravě. Brno.

NEUHÄSELOVÁ, Z. 2001: Mapa potenciální prirrozené vegetace České republiky. Praha.

NOVÁČEK, K. 2003: Rezidualita v městských souvrstvích. In: Šmejda, L., Vařeka, P. (Eds.): Sedmdesát neústupných let. Katedra archeologie, FHS ZČU v Plzni, 131-146.

OPRAVIL, E. 1964: Středověké jámy a studny. Archeologické rozhledy 16, 219-222.

ORNA, J. (Ed.) 2011: Keramická produkce města Plzně. Plzeň.

PETRÁŇ, J. et al. 1985: Dějiny hmotné kultury I. Praha.

POKORNÁ, A., DRESLEROVÁ, D., KŘIVÁNKOVÁ, D. 2011: Archaeobotanical Database of the Czech Republic, an Interim Report. Interdisciplinaria archaeologica. Natural Sciences in Archaeology II/1/2011, 49-53.

POKORNÝ, P., KOČÁR, P., JANKOVSKÁ, V., MILITKÝ, J., ZAVŘEL, P. 2002: Archaeobotany of the High Medieval town of České Budějovice. Archeologické rozhledy 54/4, 813-836.

POKORNÝ, P., KUNEŠ, P. 2009: Contributions to the European Pollen Database 5. Kožlí (S. Bohemia, Czech Republic). Grana 48, 77-78.

PROCHÁZKA, R. 1994: Brněnská stolní a kuchyňska keramika 2. poloviny 14. století - počátku 15. století. Pravěk NR̆ 4, 323-344.

PRUMMEL, W., FRISCH, H. J. 1986: A guide for distinction of species, sex and body side in bones of sheep and goat. Journal of Archaeological Science 13, 567-577.

PUNT, W., BLACKMORE, S., ClARKE, G. C. S. (Eds.) 1988: The Northwest European Pollen Flora, Vol. V. Elsevier, Amsterdam.

PUNT, W., BLACKMORE, S, HOEN, P. P., STAFFORD, P. J. (Eds.) 20032009: The Northwest European Pollen Flora, Vol. VIII, IX. Elsevier, Amsterdam.

PUNT, W., BLACKMORE, S., HOEN, P. P. (Eds.) 1995: The Northwest European Pollen Flora, Vol. VII. Elsevier, Amsterdam.

PUNT, W., BLACKMORE, S. (Eds.) 1991: The Northwest European Pollen Flora, Vol. VI. Amsterdam: Elsevier. 
PUNT, W., CLARKE, G. C. S. (Eds.) 1980-1984: The Northwest European Pollen Flora, Vol. II, Vol. III, Vol. IV. Elsevier, Amsterdam.

PUNT, W. (Ed.) 1976: The Northwest European Pollen Flora, Vol. I. Elsevier, Amsterdam.

RADOMĚRSKÝ, P., RICHTER, M. 1976: Korpus české středověké keramiky datované mincemi. Sbornik Narodniho muzea v Praze 28A, $57-171$.

REICHERTOVÁ, K. 1965: Středověka keramika ze Sezimova Ústí, Tábora a Koziho Hrádku. Archeologické studijni materiály, Vol. III. Praha.

RICHTER, M., KRAJÍC, R. 2001: Sezimovo Ústi. Archeologie středověkého poddanského města, 2. Levobřěni predměstí - archeologický výzkum 1962-1988. Archeologický ústav AV ČR, Praha.

RYBNÍČKOVÁ, E., RYBNÍČEK, K. 1985: Paleogeobotanical Evaluation of the Holocene Profile from the Rezabinec Fish-Pond. Folia Geobotanica \& Phytotaxonomica 20(4), 419-437.

SEDLÁČEK, A. 1912: Dějiny královského města Písku nad Otavou. Od žrizení královského úŕadu do dnešni doby. Písek.

SCHWEINGRUBER F. H., 1990: Microscopic Wood Anatomy; Structural variability of stems and twigs in recent and subfossil woods from Central Europe. Birmensdorf, Eidgenössische Forschungsanstalt WSL.

SILVER, I. A. 1969: The ageing of domestic animals. In: Brothwell, D., Higgs, E. S.: Science in Archaeology. Thames and Hudson, London, 283-302.

SLAVÍK, B., ŠTĚPÁNKOVÁ, J. (Eds.) 2004: Květena České republiky, Vol 7. Academia, Praha.
SLAVÍK, B., (Ed.) 1995-2000: Květena České republiky, Vol 4, 5, 6. Academia, Praha.

ŠTĚPÁNKOVÁ, J., (Ed.) 2010: Květena České republiky, Vol 8. Academia, Praha.

SMITH, D. N. 2013: Defining an indicator package to allow identification of "cesspits" in the archaeological record. Journal of Archaeological Science 40, 526-543.

SOUKUP, J. 1910: Soupis památek, 33. Politický okres písecký, 170.

ŚWIĘTA-MUSZNICKA, J., LATAŁOWA, M., BADURA, M., GOŁEMBNIK, A. 2013: Combined pollen and macrofossil data as a source for reconstructing mosaic patterns of the early medieval urban habitats e a case study from Gdańsk, N. Poland. Journal of Archaeological Science 40, 637-648.

ŠIROKÝ, R. 2000: Pitná, užitková a odpadní voda v českých městech ve středověku a raném novověku: Stav a perspektivy archeologického poznání. Památky archeologické 91/2, 345-410.

von THÜNEN, J. H. 1826: Der islolierte Staat in Beziehung auf Landwirtschaft und Nationalökonomie. Jena.

VAŘEKA, P. 1998: Proměny keramické produkce vrcholného a pozdního středověku v Čechach. Archeologické rozhledy 50, 123-137.

WINTER, Z. 1901: Život a učení na partikulárních školách v Čechách v XV. a XVI. století. Praha. 